\title{
STATIONARY CONFIGURATIONS OF POINT VORTICES
}

\author{
KEVIN ANTHONY O'NEIL
}

\begin{abstract}
The motion of point vortices in a plane of fluid is an old problem of fluid mechanics, which was given a Hamiltonian formulation by Kirchhoff. Stationary configurations are those which remain self-similar throughout the motion.

Results of two types are presented. Configurations which are in equilibrium or which translate uniformly are counted using methods of algebraic geometry, which establish necessary and sufficient conditions for existence.

Relative equilibria (rigidly rotating configurations) which lie on a line are studied using a topological construction applicable to other power-law systems. Upper and lower bounds for such configurations are found for vortices with mixed circulations.

Arrangements of three vortices which collide in finite time are well known. One-dimensional families of such configurations are shown to exist for more than three vortices.

Stationary configurations of four vortices are examined in detail.
\end{abstract}

The motion of point vortices in a plane of fluid is an old problem of fluid mechanics, which was given a Hamiltonian formulation by Kirchhoff. Stationary configurations are those which remain self-similar throughout the motion, and are of considerable physical interest.

It is known that a configuration of vortices in equilibrium must have total vortex angular momentum 0 . A converse is proved, namely that for almost every choice of circulations with zero vortex angular momentum, there are exactly $(n-2)$ ! equilibrium configurations. A similar statement is proved for rigidly translating configurations with total circulation zero. The proofs involve ideas from algebraic geometry.

Relative equilibria (rigidly rotating configurations) were studied by Palmore in the case of positive circulations. Upper and lower bounds for the number of collinear relative equilibria for arbitrary circulations are obtained by means of a topological construction which is applicable to other power-law systems.

Arrangements of three vortices which collide in finite time are well known. One-dimensional families of such configurations are shown to exist for $n$ vortices, $n \geqslant 3$.

Stationary configurations of four vortices are examined in detail.

\section{Chapter 0. INTRODUCTION}

The motion of point vortices in the plane is an old problem in fluid mechanics. It was first given a Hamiltonian formulation by Kirchhoff (1876), who proved that $n$ vortices with distinct positions $\left(x_{i}, y_{i}\right)(i=1, \ldots, n)$ in the $(x, y)$ plane, and

Received by the editors July 31, 1985 and, in revised form, June 13, 1986.

1980 Mathematics Subject Classification (1985 Revision). Primary 76C05; Secondary 70 H05. 
nonzero real circulations $\kappa_{i}$ satisfy

$$
\kappa_{i} \frac{d x_{i}}{d t}=\frac{\partial H}{\partial y_{i}}, \quad \kappa_{i} \frac{d y_{i}}{d t}=-\frac{\partial H}{\partial x_{i}}
$$

where

$$
H=-\frac{1}{2} \sum \kappa_{i} \kappa_{j} \ln \left[\left(x_{i}-x_{j}\right)^{2}+\left(y_{i}-y_{j}\right)^{2}\right] .
$$

Equations (1) can be given exact Hamiltonian form by making the substitutions

$$
q_{i}=x_{i}\left(\sqrt{\left|\kappa_{i}\right|}\right), \quad p_{i}=y_{i}\left(\kappa_{i} / \sqrt{\left|\kappa_{i}\right|}\right) .
$$

Then (1) becomes the usual Hamiltonian equations with canonical coordinates $q_{i}$ and momenta $p_{i}$ :

$$
\frac{d q_{i}}{d t}=\frac{\partial H}{\partial p_{i}}, \quad \frac{d p_{i}}{d t}=-\frac{\partial H}{\partial q_{i}} .
$$

By performing the differentiations indicated in (1), one finds the velocity of the $i$ th vortex:

$$
\frac{d x_{i}}{d t}=-\sum_{j}^{\prime} \frac{\kappa_{j}}{d_{i j}^{2}}\left(y_{i}-y_{j}\right), \quad \frac{d y_{i}}{d t}=\sum_{j}^{\prime} \frac{\kappa_{j}}{d_{i j}^{2}}\left(x_{i}-x_{j}\right),
$$

where

$$
d_{i j}^{2}=\left(x_{i}-x_{j}\right)^{2}+\left(y_{i}-y_{j}\right)^{2}
$$

and the prime on the summation indicates omission of the term with $l=j$. The position of the $i$ th vortex may be given by the complex number $z_{l}=x_{l}+i y_{l}$; then we find that the velocity $V_{l}$ of the $l$ th vortex is

$$
V_{l}=\frac{d z_{l}}{d t}=i \sum^{\prime} \frac{\kappa_{j}}{d_{l j}^{2}}\left(z_{l}-z_{j}\right)=i \sum^{\prime} \frac{\kappa_{j}}{\overline{z_{l}-z_{j}}} .
$$

Definition 0.1. The local dynamical system of $n$ point vortices in the plane, with positions $z_{l}$ and circulations $\kappa_{l}(l=1, \ldots, n)$ is given by the flow of the vector field

$$
V=\left(V_{1}, \ldots, V_{n}\right), \quad V_{l}=(\sqrt{-1}) \sum_{j}^{\prime} \frac{\kappa_{j}}{\overline{z_{l}-z_{j}}}
$$

on the set $\left\{z=\left(z_{1}, \ldots, z_{n}\right) \in \mathbf{C}^{n} \mid z_{l} \neq z_{j} \forall l \neq j\right\}$. The point $\kappa=\left(\kappa_{1}, \ldots, \kappa_{n}\right) \in \mathbf{R}^{n}$ is called the choice of circulations, and each $\kappa_{l}$ is nonzero unless otherwise noted.

Historically, particular attention has been given to the following class of vortex motions.

Definition 0.2. $\left(z_{1}, \ldots, z_{n}\right)$ is stationary if there is a constant $\omega \in \mathbf{C}$ so that the velocities and positions satisfy

$$
V_{l}-V_{j}=\omega\left(z_{l}-z_{j}\right), \quad \forall l, j
$$

The geometric meaning of stationary is that the shape of the arrangement of points $z_{1}, \ldots, z_{n}$ in the plane is preserved by the motion. One sees this by checking that the ratio of any two intervortex distances $d_{l j} / d_{r s}$ is independent of time. 
Proposition 1.1.4 proves that $z$ is stationary iff it is one of the following types of motion.

DeFINITION 0.3. (a) $z$ is an equilibrium if $V_{1}=\cdots=V_{n}=0$.

(b) $z$ is rigidly translating if $V_{1}=\cdots=V_{n}=V \neq 0$ for some $V \in \mathbf{C}$. (The vortices are said to move with common velocity $V$.)

(c) $z$ is a relative equilibrum if there exist constants $0 \neq \lambda \in \mathbf{R}, z_{0} \in \mathbf{C}$, so that $V_{l}=i \lambda\left(z_{l}-z_{0}\right) \forall l$.

(d) $z$ is a collapse if there exist constants $\omega, z_{0} \in \mathbf{C}$ so that $V_{l}=\omega\left(z_{l}-z_{0}\right) \forall l$, and $\operatorname{Re}(\omega) \neq 0$.

It is easy to see that if $z$ is stationary then so is any other geometrically similar arrangement of vortices $z^{\prime}$, that is, $z^{\prime}$ differs from $z$ only by translation, rotation, and change of scale.

Definition 0.4. $z$ is equivalent to $z^{\prime}$ if for some $a, b \in \mathbf{C}$ with $b \neq 0, z_{l}^{\prime}=$ $b\left(z_{l}+a\right) \forall l$. The equivalence class $[z]$ of $z$ is called a configuration. The configuration space is the set

$$
N=\left\{\left[\left(z_{1}, \ldots, z_{n}\right)\right] \mid z_{l} \in \mathbf{C} ; z_{l} \neq z_{j} \forall l \neq j\right\} .
$$

The property of being stationary is well defined on the configuration space. It follows from Proposition 1.1.4 that if $z$ is stationary then $[z]$ is independent of time; the motion is "stationary" in configuration space.

In Chapter 1, configuration space is identified in a natural way with a subset of $(n-2)$-dimensional complex projective space, $\mathbf{P}^{n-2}$. If the positions $z_{1}, \ldots, z_{n}$ are collinear then configuration space is a corresponding subset of real projective space $\mathbf{R} P^{n-2}$.

Main results. Define the total circulation to be $\sigma=\sum_{l} \kappa_{l}$, and the (total) vortex angular momentum to be $L=\sum_{l<j} \kappa_{l} \kappa_{j}$. It is well known (see Lemma 1.2.1) that if $z$ is an equilibrium then $L=0$, and if $z$ is rigidly translating then $\sigma=0$. For the 3-vortex system, the converse is also true: There are always equilibria or rigidly translating configurations when $\kappa$ satisfies the appropriate condition. For larger $n$, almost nothing is known about these configurations; all that appears in the literature is a highly symmetrical class of equilibria described by Havelock (1931). The following converse to the above necessary conditions is proved in Chapter 5 .

THEOREM 5.1.1 (RESP., 5.2.1). For almost every $\kappa=\left(\kappa_{1}, \ldots, \kappa_{n}\right)$ satisfying $L=0$ (resp. $\sigma=0)$, there are exactly $(n-2)$ ! (resp. $(n-1)$ !) equilibrium (resp. rigidly translating) configurations.

The almost-everywhere condition is made precise in Chapter 5.

These theorems are proved by considering the set of stationary configurations with $\omega=0$ as a subvariety of a certain space. In order to show this, lemmas of a technical nature are proved in Chapter 3 which allow us to separate this set from the "diagonal" (see Definition 1.1.6). The projection from the stationary configurations to the choice of circulations is an étale map. The number of stationary configurations is found by a repeated application of the implicit function theorem. 
Relative equilibrum configurations are so called because the motion vanishes in some rotating reference frame. The study of such configurations was termed "vortex statics" by Kelvin (1910), who, with Thompson (1883) found relative equilibria of identical vortices. Havelock (1931) studied vortices arranged in two concentric rings. If all circulations have the same sign, relative equilibria are the only stationary configurations possible. Palmore (1982) investigated this case and found that many relative equilibria must occur.

Aside from special solutions found in Havelock and in Novikov and Sedov (1979), the only relative equilibria known to exist when the $\kappa_{i}$ have mixed signs are those found by analytical continuation, from the all-positive case to one or more weak negative circulations.

In Chapters 6 and 7, the projective-space setting is exploited to prove continuation-type theorems which hold, not for "sufficiently weak" negative circulations, but for circulations satisfying a finite number of inequalities. The tool used is the degree of a component of configuration space, given by the topological degree of a map of spheres. The useful property of the degree is established in Propositions 6.3.3 and 7.2.2, and lower bounds for the number of collinear relative equilibria are produced in Theorems 6.3.5 and 7.2.3. Chapter 6 concludes with Theorem 6.5.1, giving the best possible upper bound for the number of collinear relative equilibria.

The final class of stationary configurations is the collapse configuration. Collapses of 3 vortices were demonstrated by Gröbli (1877); Synge (1949) proved that the collapse configurations form a circle in configuration space. Again, there is nothing known for higher $n$ besides the special solutions found in Novikov and Sedov (1979).

In Chapter 7 it is proved that almost every collinear relative equilibrium with $L=0$ lies on a family of collapsing configurations, for every $n \geqslant 3$. Thus, vortex collapse is not merely a phenomenon of simple vortex systems.

Chapter 8 is devoted to the 4-vortex system, where an explicit formula for equilibria is derived. Collinear relative equilibria are shown to exist for almost every choice of circulations having $L=0$; these results (Theorem 8.3.3) are presented in a graph in Figure 6. Finally, Theorem 8.4 .1 proves that for every choice of circulations having $\sigma=0$, there are at least four relative equilibria.

\section{Chapter 1. Stationary CONFigurations}

1.1. The $N$-vortex system. Given $n$ vortices in the plane $\mathbf{C}$ with distinct positions $z_{1}, \ldots, z_{n}$ and circulations $\kappa_{1}, \ldots, \kappa_{n}$, each $\kappa_{l} \in \mathbf{R}-\{0\}$, then the velocity of the $l$ th vortex is given by

$$
V_{l}=i \sum_{l \neq j} \frac{\kappa_{j}}{\overline{z_{l}-z_{j}}}
$$

(See the Introduction, or Sommerfeld (1964), p. 158, Lamb (1932), p. 219, Batchelor (1967), p. 530.) 
DEFINITION 1.1.1. The following quantities are defined:

$\begin{array}{ll}\text { Total circulation } & \sigma=\sum \kappa_{l} \\ \text { Total vortex angular momentum } & L=\sum \kappa_{l<j} \kappa_{j} \\ \text { Moment of vorticity } & M=\sum \kappa_{l} z_{l} \\ \text { Center of vorticity } & C=M / \sigma \\ \quad(\text { when } \sigma \neq 0) & \\ \text { Size } & S=\sum \kappa_{l}\left|z_{l}\right|^{2}\end{array}$

LEMMA 1.1.1. The following identities hold:

(I1) $\sum \kappa_{l} V_{l}=0$,

(I2) $\sum \kappa_{l} \bar{z}_{l} V_{l}=i L$.

Proof. $\sum \kappa_{l} V_{l}=\sum_{l \neq j} \kappa_{l} \kappa_{j} /\left(\overline{z_{l}-z_{j}}\right)=0$, since the $(l j)$ term cancels the $(j l)$ term. In (12),

$$
\sum \kappa_{l} \bar{z}_{l} V_{l}=i \sum_{l \neq j} \kappa_{l} \kappa_{j} \frac{\bar{z}_{l}}{\overline{z_{l}-z_{j}}}=i \sum_{l \neq j} \kappa_{l} \kappa_{j} \frac{\bar{z}_{j}}{\overline{z_{j}-z_{l}}},
$$

the latter by reindexing. Take the average of the two:

$$
\sum \kappa_{l} \bar{z}_{l} V_{l}=\frac{i}{2} \sum_{l \neq j} \kappa_{l} \kappa_{j} \frac{\overline{z_{l}-z_{j}}}{\overline{z_{l}-z_{j}}}=\frac{i}{2} \sum_{l \neq j} \kappa_{l} \kappa_{j}=i L,
$$

as desired.

The translate by $a$ of $\left(z_{1}, \ldots, z_{n}\right)$ is the point $\left(z_{1}+a, \ldots, z_{n}+a\right)$. Note that since $\sum \kappa_{l}\left(z_{l}+a\right)=\sum \kappa_{l} z_{l}+\sigma a, M$ is translation-invariant iff $\sigma=0$. It is similar to check that $S$ is translation-invariant iff $M=0$ and $\sigma=0$. Also, since $\sigma^{2}-2 L=$ $\sum \kappa_{l}^{2}>0$, the quantities $\sigma$ and $L$ cannot vanish simultaneously.

Identity (I1) says that $M$ is preserved by the motion. If two complex numbers are viewed as vectors in $E^{2}$, with standard inner product $\langle$,$\rangle , then \langle u, v\rangle=\operatorname{Re}(\bar{u} v)$. Thus,

$$
\frac{d}{d t}(S)=2 \sum \kappa_{l}\left\langle z_{l}, V_{l}\right\rangle=2 \operatorname{Re}\left(\sum \kappa_{l} \bar{z}_{l} V_{l}\right)=0
$$

So $S$ is preserved by the motion.

Recall the following definitions.

Definition 1.1.2. $z=\left(z_{1}, \ldots, z_{n}\right)$ is called stationary if there is a constant $\omega \in \mathbf{C}$ so that $V_{l}-V_{j}=\omega\left(z_{l}-z_{j}\right) \forall l, j$.

Definition 1.1.3. (a) $z$ is an equilibrium if $V_{1}=\cdots=V_{n}=0$.

(b) $z$ is rigidly translating if $V_{1}=\cdots=V_{n} \neq 0$.

(c) $z$ is a relative equilibrium if $\exists \lambda \in \mathbf{R}, z_{0} \in \mathbf{C}$, with $0 \neq \lambda$, so that $V_{l}=$ $i \lambda\left(z_{l}-z_{0}\right) \forall l$.

(d) $z$ is collapsing if $\exists \omega \in \mathbf{C}, z_{0} \in \mathbf{C}$ such that $V_{l}=\omega\left(z_{l}-z_{0}\right) \forall l$, and $\operatorname{Re}(\omega) \neq 0$.

Proposition 1.1.4. $z$ is stationary iff it satisfies one of the conditions given in Definition 1.1.3. 
Proof. If $z$ is as in (a)-(d), then $z$ is clearly stationary. So assume $V_{l}-V_{j}=$ $\omega\left(z_{l}-z_{j}\right) \forall l, j$. If $\omega=0$ then $z$ is an equilibrium or is rigidly translating. Next, suppose $\omega \neq 0$. First, assume $\sigma \neq 0$. From $V_{l}-V_{j}=\omega\left(z_{l}-z_{j}\right)$, we find

$$
\sigma V_{l}=\sum_{j} \kappa_{j}\left(V_{l}-V_{j}\right)=\omega\left(\sigma z_{l}-M\right) .
$$

Thus $V_{l}=\omega\left(z_{l}-C\right)$, as in (c) or (d) above.

If $\sigma=0$, pick $z_{0}=z_{l}-V_{l} / \omega$. Since $V_{l}-\omega z_{l}=V_{j}-\omega z_{j}$ for all $l, j$, the point $z_{0}$ does not depend on the choice of index. Moreover $V_{l}=\omega\left(z_{l}-z_{0}\right)$, and the proposition is proved.

We thus see that Definition 1.1 .3 categorizes all stationary points.

DeFINITION 1.1.5. $z, z^{\prime} \in \mathbf{C}^{n}$ are equivalent if $z_{l}^{\prime}=c\left(z_{l}+a\right) \forall l$, for some $c$, $a \in \mathbf{C}$, with $c \neq 0$. The equivalence class of $z$ is denoted by $[z]$ and called a configuration. The configuration space is the set

$$
N=\left\{\left[z_{1}, \ldots, z_{n}\right] \mid z_{l} \in \mathbf{C} ; z_{l} \neq z_{j} \forall l \neq j\right\} .
$$

Note that $z \sim z^{\prime}$ if the positions of the vortices differ only by translation, rotation, and scale. It is convenient to identify the configuration space $N$ with a certain subset of complex projective space. If $\sigma \neq 0$, we may take as the representative of an equivalence class the unique translate having $M=0$. Then $N$ is the following subset of $\mathbf{P}^{n-1}$ :

$$
\left\{\left[z_{1}, \ldots, z_{n}\right] \in \mathbf{P}^{n-1} \mid M=0 \text { and } z_{l} \neq z_{j} \forall l \neq j\right\} .
$$

When $\sigma=0, M$ is translation invariant. We may take as representative the translate having $z_{1}=0$. Then $N$ is identified with

$$
\left\{\left[z_{1}, \ldots, z_{n}\right] \in \mathbf{P}^{n-1} \mid z_{1}=0 ; z_{l} \neq z_{j} \forall l \neq j\right\} .
$$

Definition 1.1.6. The diagonal $\Delta$ is the collection of hyperplanes in $\mathbf{P}^{n-1}$ where two or more vortices coincide:

$$
\Delta=\left\{\left[z_{1}, \ldots, z_{n}\right] \in \mathbf{P}^{n-1} \mid z_{l}=z_{j} \text { for some } l \neq j\right\} .
$$

From the above, we see that $N=\mathbf{P}^{n-2}-\Delta$. From Proposition 1.1.4 one concludes that for stationary $z,[z]$ is independent of time. Thus the motion is "stationary" in projective space.

If the vortices are collinear, we may take $\operatorname{Im}\left(z_{l}\right)=0 \forall l$. The configuration space is then the corresponding subset of real projective space $\mathbf{R} P^{n-2}$.

1.2. Reduction of the problem. The identities of Lemma 1.1.1 can be used to reduce the number of equations needed to define stationary configurations, as well as to find necessary conditions for them.

LEMMA 1.2.1. Every equilibrium has circulations satisfying $L=0$; every rigidly translating configuration has circulations satisfying $\sigma=0$.

Proof. If $V_{1}=\cdots=V_{n}=0$, (I2) becomes $i L=0$. If $V_{1}=\cdots=V_{n}=V \neq 0$, where $V$ is the common velocity, then (I1) reads $0=\sigma V$ so $\sigma=0$.

REMARK. Chapter 5 is devoted to proving a converse to this lemma. 
Proposition 1.2.2. Let $L=0$. If $V_{l}=0$ for $(n-2)$ choices of index $l$, then $V_{l}=0 \forall l$.

Proof. The ordering of the vortices is unimportant; assume $V_{3}=\cdots=V_{n}=0$. By Lemma 1.1.1,

(I1) $\kappa_{1} V_{1}+\kappa_{2} V_{2}=0$.

(I2) $\kappa_{1} \bar{z}_{1} V_{1}+\kappa_{2} \bar{z}_{2} V_{2}=0$.

Since $z_{1} \neq z_{2}$, the homogeneous linear system has unique solution $V_{1}=V_{2}=0$. This proves the proposition.

Proposition 1.2.3. Let $\sigma=0$. If $V_{l}=i L / \bar{M}$ for $(n-2)$ choices of index $l$, then $V_{l}=i L / \bar{M} \forall l$.

Proof. Again, we may assume $V_{3}=\cdots=V_{n}=i L / \bar{M}=V$.

(I1) $\kappa_{1}\left(V_{1}-V\right)+\kappa_{2}\left(V_{2}-V\right)=\sum \kappa_{l}\left(V_{l}-V\right)=0$.

(I2) $\kappa_{1} \bar{z}_{1}\left(V_{1}-V\right)+\kappa_{2} \bar{z}_{2}\left(V_{2}-V\right)=\sum \kappa_{l} \bar{z}_{l}\left(V_{l}-V\right)=i L-V \bar{M}=0$.

Since $z_{1} \neq z_{2}$, the result follows.

These propositions show that finding equilibrium configurations is equivalent to finding the common zeros of $n-2$ homogeneous rational functions of $z_{l}$, namely, $\bar{V}_{l}$, on $\mathbf{P}^{n-2}$; finding rigidly translating configurations is equivalent to finding the common zeros of $n-2$ rational functions $\bar{V}_{l}+i L / M$ on $\mathbf{P}^{n-2}$. These functions have poles in the set $\Delta$, so the stationary configurations are the common zeros of $n-2$ homogeneous polynomials - the numerators of the rational functions-in $\mathbf{P}^{n-2}-\Delta$. This is the setting for Bezout's theorem, and this problem is addressed in Chapter 5 .

Next consider the stationary configurations with $\omega \neq 0$. The following lemma establishes some necessary conditions.

Lemma 1.2.4. Suppose $V_{l}-V_{j}=\omega\left(z_{l}-z_{j}\right) \forall l, j$, and $\omega \neq 0$. Then

(a) If $\sigma=0$, then $M=0$.

(b) If $M=0$, then for any $\sigma$, iL $=\omega S$. In particular, if $L \neq 0$ then $\omega=i L / S$ while if $L=0$ then $S=0$.

(c) If $L \neq 0$ then $\operatorname{Re}(\omega)=0$, and $z$ is a relative equilibrium.

Proof. From $V_{l}-V_{j}=\omega\left(z_{l}-z_{j}\right)$ we find that

$$
\sigma V_{l}=\sum_{j} \kappa_{j}\left(V_{l}-V_{j}\right)=\omega\left(\sigma z_{l}-M\right) \text {. }
$$

If $\sigma=0$, then $0=\omega M$, proving (a). Next,

$$
V_{l} \bar{M}-i L=\sum_{j} \kappa_{j} \bar{z}_{j}\left(V_{l}-V_{j}\right)=\omega \sum_{j} \kappa_{j} \bar{z}_{j}\left(z_{l}-z_{j}\right)=\omega\left(z_{l} \bar{M}-S\right) .
$$

Setting $M=0$ completes (b). Lastly, (c) is a consequence of (b).

Proposition 1.2.5. Assume $L \neq 0, \sigma \neq 0$, and $M=0$. If $V_{l}=i(L / S) z_{l}$ for $n-2$ choices of index $l$, then $V_{l}=i(L / S) z_{l} \forall l$, and $z$ is a relative equilibrium.

Proof. Fix $\omega=i L / S$. We may assume $V_{l}=\omega z_{l}, l=3, \ldots, n$. Use Lemma 1.1.1:

$$
\kappa_{1}\left(V_{1}-\omega z_{1}\right)+\kappa_{2}\left(V_{2}-\omega z_{2}\right)=\sum \kappa_{l}\left(V_{l}-\omega z_{l}\right)=0 .
$$


(I2)

$$
\kappa_{1} \bar{z}_{1}\left(V_{1}-\omega z_{1}\right)+\kappa_{2} \bar{z}_{2}\left(V_{2}-\omega z_{2}\right)=\sum \kappa_{l} \bar{z}_{l}\left(V_{l}-\omega z_{l}\right)=i L-\omega S=0 .
$$

Since $z_{1} \neq z_{2}$, the proposition follows.

Proposition 1.2.6. Let $\sigma=0$. If $V_{1}-V_{l}=(i L / S)\left(z_{1}-z_{l}\right)$ for $n-3$ choices of index $l$, and $M=0$, then $V_{1}-V_{l}=i(L / S)\left(z_{1}-z_{l}\right) \forall l$, and $z$ is a relative equilibrium.

Proof. Let $\omega=i L / S$, and write $D_{l}=\left(V_{1}-V_{l}\right)-\omega\left(z_{1}-z_{l}\right), 2 \leqslant l \leqslant n$. We may assume $D_{4}=\cdots=D_{n}=0$. Then

$$
\kappa_{2} D_{2}+\kappa_{3} D_{3}=\sum \kappa_{l}\left[V_{1}-V_{l}-\omega\left(z_{1}-z_{l}\right)\right]=\sigma\left(V_{1}-\omega z_{1}\right)+\omega M=0 .
$$

$$
\begin{aligned}
\kappa_{2} \bar{z}_{2} D_{2}+\kappa_{3} \bar{z}_{3} D_{3} & =\sum \kappa_{l} \bar{z}_{l}\left[V_{1}-V_{l}-\omega\left(z_{1}-z_{l}\right)\right] \\
& =\bar{M}\left(V_{1}-\omega z_{1}\right)-i L+\omega S=0 .
\end{aligned}
$$

Since $z_{1} \neq z_{2}$, we conclude that $D_{2}=D_{3}=0$, completing the proof.

Proposition 1.2.7. Suppose $L=0$ and $M=0$. If $V_{1} z_{l}-V_{l} z_{1}=0$ for $n-3$ values of index $l$, and $S=0$, then $V_{1} z_{l}-V_{l} z_{1}=0 \forall l$, and $z$ is a relative equilibrium or collapse.

Proof. Assume $D_{l}=V_{1} z_{l}-V_{l} z_{1}=0$ for $l=4, \ldots, n$.

$$
\kappa_{2} D_{2}+\kappa_{3} D_{3}=\sum \kappa_{l}\left(V_{1} z_{l}-V_{l} z_{1}\right)=V_{1} M=0
$$

$$
\kappa_{2} \bar{z}_{2} D_{2}+\kappa_{3} \bar{z}_{3} D_{3}=\sum \kappa_{l} \bar{z}_{l}\left(V_{1} z_{l}-V_{l} z_{1}\right)=V_{1} S-z_{1}(i L)=0 .
$$

Since $z_{3} \neq z_{2}$, the proposition holds.

The above facts reduce the number of equations needed to find stationary configurations having $\omega \neq 0$.

If $L \neq 0$ and $\sigma \neq 0$, it suffices to find the common zeros of $n-2$ functions of the form $\left(V_{l}-i(L / S) z_{l}\right)$ in $\mathbf{P}^{n-2}$. These are not rational functions of $z$ since they also involve $\bar{z}$. However, they are complex-valued and one would expect the solution set in $\mathbf{P}^{n-2}$ to have dimension 0 .

When $\sigma=0$, one of the above functions may be replaced by the first-degree polynomial $M$. In other words, we may restrict our domain to the hyperplane $\mathbf{P}^{n-3}$ in $\mathbf{P}^{n-2}$ having $M=0$. Again one expects the solution set to have dimension 0 .

When $L$ vanishes, however, relative equilibria and collapse configurations are given by the common zeros of $n-3$ complex valued functions $V_{1} z_{1}-V_{1} z_{1}$ and the real valued function $S$. Thus one expects the solution set to have dimension 1 . This is found to be the case in Chapter 7 . 
1.3. Nondegeneracy. To reduce repetition in the sequel, we make a definition.

DEFINITION 1.3.1. The defining functions of stationary configurations are as follows:

(a) for equilibria, $f_{l}=\bar{V}_{l+2}, l=1, \ldots, n-2$;

(b) for rigidly translating configurations, $f_{l}=\bar{V}_{l+2}+i L / M, l=1, \ldots, n-2$;

(c) for relative equilibria (with $L \neq 0, \sigma \neq 0$ ), $f_{l}=V_{l+2}-i L / S z_{l+2}, l=1, \ldots$, $n-2$;

(d) relative equilibria $(\sigma=0): f_{1}=M ; f_{l}=\left(V_{1}-V_{l+2}\right)-i L / S\left(z_{1}-z_{l+2}\right), l=$ $2, \ldots, n-2$

(e) relative equilibria and collapse, $L=0: f_{1}=S ; f_{l}=V_{1} z_{l+2}-V_{l+2} z_{1}, \quad l=$ $2, \ldots, n-2$.

Thus by $\$ 1.2$ the stationary configurations are the zero sets of the respective defining functions.

We obtain an affine neighborhood $U$ of a point $z$ in $\mathbf{P}^{n-2}-\Delta$ as follows. Some local choice of coordinates (for instance, euclidean coordinates on $\mathbf{P}^{n-1}$ ) gives a neighborhood $U \subset \mathbf{C}^{n-2}$ of a representative $z \in \mathbf{C}^{n}$. Thus the defining functions are locally functions of these coordinates $\tilde{z}_{1}, \ldots, \tilde{z}_{n}$. $U$ is homeomorphic to a neighborhood of $z$ found by keeping two positions fixed (e.g., $z_{1}$ and $z_{2}$ ) and allowing the remaining $z_{i}$ to range through a suitably small neighborhood. So we may think of the defining functions as locally being functions of $n-2$ of the positions $z_{l}$.

In Chapter 4 we will make use of the implicit function theorem. The next definition is given with this in mind.

Definition 1.3.2. A stationary configuration $[z] \in N$ is nondegenerate if there is a neighborhood $U \in \mathbf{C}^{n-2}$ of a representative $z$ of $[z]$ so that the gradients of the real and imaginary parts of the defining functions $f_{l}$ are independent at $z$.

For equilibria and rigidly translating configurations, the defining functions $f_{l}$ are analytic in a neighborhood of the stationary configuration. So $[z]$ is nondegenerate iff the matrix $\partial f_{l} / \partial z_{j}$ is nonsingular at $z$; the $z_{j}$ are any local coordinates, e.g., $z_{3}, \ldots, z_{n}$.

For relative equilibria with $L \neq 0$, the defining functions are not analytic. Let $z_{l}=x_{l}+i y_{l}$. Then nondegeneracy of $[z]$ is equivalent to the nonsingularity of the $(2 n-4)$ by $(2 n-4)$ real Jacobian matrix of the functions $\left\{\operatorname{Re}\left(f_{l}\right), \operatorname{Im}\left(f_{l}\right)\right\}$.

Should the configuration be collinear, the matrix "splits":

$$
0=\frac{\partial}{\partial x_{j}}\left(\operatorname{Re} f_{l}\right)=\frac{\partial}{\partial y_{j}}\left(\operatorname{Im} f_{l}\right)
$$

so that nondegeneracy is equivalent to the nonvanishing at $z$ of the determinants

$$
\left|\frac{\partial}{\partial x_{j}}\left(\operatorname{Re} f_{l}\right)\right| ; \quad\left|\frac{\partial}{\partial y_{j}}\left(\operatorname{Im} f_{l}\right)\right| .
$$

We now find a rational function of $z$ which is nonzero at a collinear configuration whenever the determinants of (1) are. Write

$$
d f_{l}=p_{l j} d z_{j}+q_{l j} d \bar{z}_{j}=\left(p_{l j}+q_{l j}\right) d x_{j}+\left(p_{l j}-q_{l j}\right) i d y_{j}
$$


where $p_{l j}, q_{l j}$ are rational functions of $z$ and $\bar{z}$. Let $\tilde{p}_{l j}, \tilde{q}_{l j}$ be the same functions but "forgetting" conjugation. Then the rational function of $z$ alone, $\left|\tilde{p}_{l j}+\tilde{q}_{l j}\right|$. $\left|\tilde{p}_{l j}-\tilde{q}_{l j}\right|$, agrees with the product of the determinants in (1) on collinear configurations.

Finally, for case (e) of Definition 1.3.1, there are only $2 n-5$ real vectors to consider since $S$ is real valued. So nondegeneracy is not expressed using a determinant. To restrict attention to relative equilibria only, we may add the condition that $\operatorname{Re}(\omega)=0$. Then, as in the preceding paragraph, we can find a rational function of $z$, the nonvanishing of which implies nondegeneracy at a collinear relative equilibrium.

We may sometimes wish to restrict our implicit function theorem argument to real projective space. This motivates the following definition.

DEFINITION 1.3.3. A collinear stationary configuration $[z]$ is nondegenerate as a collinear configuration (or nondegenerate in $\mathbf{R} P^{n-2}$ ) if the first determinant of (1) is not zero at $z$.

It is easy to check that this determinant is not zero if $\left|\partial \tilde{f}_{l} / \partial z_{j}\right| \neq 0$, where the $\tilde{f}_{l}$ are obtained from $f_{l}$ by "forgetting" conjugation.

The advantage of having such rational functions of $z$ will become evident in Chapters 5-7. In that setting stationary configurations appear as points on a quasi-projective variety in a suitable product space. The rational functions are then nonzero on all but a subvariety of codimension one, so that almost every stationary configuration is nondegenerate.

1.4. Relative equilibria as critical points. In Palmore (1982) it is proved that the critical points of the Hamiltonian $H$ restricted to the subset of $\mathbf{C}^{n}$ where $M=0$ and $S=$ constant are exactly the relative equilibria. For completeness, we repeat this calculation here.

Recall that $H=-\frac{1}{2} \sum_{l<j} \kappa_{l} \kappa_{j} \ln \left|z_{l}-z_{j}\right|^{2}$. The derivative $D H$ acts on a tangent vector $v=\left(v_{1}, \ldots, v_{n}\right)$ at a point $z \in \mathbf{C}^{n}$ as follows:

$$
D H(v)=-\sum_{l<j} \frac{\kappa_{l} \kappa_{j}}{\left|z_{l}-z_{j}\right|^{2}}\left\langle z_{l}-z_{j}, v_{l}-v_{j}\right\rangle
$$

where $\langle$,$\rangle is the usual scalar product. Rearranging,$

$$
\begin{aligned}
D H(v) & =-\sum_{l \neq j} \frac{\kappa_{l} \kappa_{j}}{\left|z_{l}-z_{j}\right|^{2}}\left\langle z_{l}-z_{j}, v_{l}\right\rangle \\
& =-\sum_{l} \kappa_{l}\left\langle\sum_{j}^{\prime} \frac{k_{j}}{\overline{z_{l}-z_{j}}}, v_{l}\right\rangle \\
& =-\sum_{l} \kappa_{l}\left\langle\frac{V_{l}}{i}, v_{l}\right\rangle .
\end{aligned}
$$

Thus $z$ is a critical point of $H$ iff it is an equilibrium. Now, $z$ is a critical point of $H$ restricted to a level surface of the function $S$ iff $\operatorname{grad} H$ is proportional to $\operatorname{grad} S$, that is,

$$
V_{l}=i \lambda z_{l}
$$


for some $\lambda \in \mathbf{R}$. This is exactly the definition of a relative equilibrium (or equilibrium, if $\lambda=0$ ).

Now define the real-valued function

$$
\tilde{H}=-\frac{1}{2} \sum_{l<j} \kappa_{l} \kappa_{j} \ln \left[\frac{\left|z_{l}-z_{j}\right|^{2}}{|S|}\right]=H+\frac{1}{2} L \ln |S| .
$$

This function is convenient because it is well defined on the subset of $\mathbf{P}^{n-2}-\Delta$ where $S \neq 0$.

REMARK. Although it is not important in the sequel, the functions $H$ and $\tilde{H}$ define the same Hamiltonian dynamical system, as is easily checked by comparing the partial derivatives in equations (1) of the Introduction.

It is easy to see that the critical points of $\tilde{H}$ are exactly the equilibria and relative equilibra:

$$
D \tilde{H}(v)=D H(v)+\frac{1}{2} \frac{L}{S} D S(v)=-\sum \kappa_{l}\left\langle\frac{V_{l}}{i}-\frac{L}{S} z_{l}, v_{i}\right\rangle .
$$

Thus the critical points are those which satisfy $(*)$ above, with $\lambda=L / S$. This is a necessary condition when $L \neq 0$. If $L=0$, then $H=\tilde{H}$ and the relative equilibria are the critical points of $H$ restricted to the set $S=0$.

If $\sigma=0$, we know from Lemma 1.1.6 that relative equilibria have $M=0$. This is not the entire configuration space, but it turns out that if $\tilde{H}$ is restricted to the set $M=0$, then the critical points are exactly the relative equilibria: critical points satisfy

$$
V_{l}=i(L / S)\left(z_{l}-p\right)
$$

where $p=(S / L)\left(\mu_{1}+i \mu_{2}\right)$ for some Lagrange multipliers $\mu_{1}, \mu_{2}$. This observation is used in $\S 8.4$.

\section{Chapter 2. The 3-VORTEX SYSTEM}

This chapter contains two lemmas concerning the existence of 3-vortex stationary configurations which are needed in Chapter 5.

LEMMA 2.1. If $\left(\kappa_{1}, \kappa_{2}, \kappa_{3}\right)$ satisfy $L=0$ then there is exactly one nondegenerate equilibrium configuration $\left[z_{1}, z_{2}, z_{3}\right]=\left[0,1,-\kappa_{3} / \kappa_{2}\right]$. Moreover, for such configurations, the meromorphic function

$$
V(z)=i \sum_{l} \kappa_{l} /\left(z-z_{l}\right)
$$

has exactly two simple zeros.

Proof. By Proposition 1.2.2, $z$ is an equilibrium iff

$$
0=i \bar{V}_{1}=\kappa_{2}+\kappa_{3} / z_{3} \text {. }
$$

So the only equilibrium configuration is as stated. Nondegeneracy is given by the nonvanishing of a single derivative, and is easily checked.

The function $V(z)$ is rational with a quadratic polynomial in the numerator. It is trivial to check that its discriminant is negative, proving the existence of two simple zeros of $V$. 
LEMMA 2.2. If $\left(\kappa_{1}, \kappa_{2}, \kappa_{3}\right)$ satisfy $\sigma=0$ then the only rigidly translating configurations are the two equilateral configurations. If $\kappa=(1,1,-2)$ then these solutions are nondegenerate and the function $V(z)-i L / \bar{M}$ has exactly three simple zeros.

Proof. By Lemma 1.1.2, $z$ is rigidly translating iff the single equation $V_{1}-V_{2}=0$ is satisfied. Set $z=\left(0,1, z_{3}\right)$; the above equation reduces to $1 /\left(1-z_{3}\right)+1 / z_{3}=1$.

The only solutions are $z_{3}=\frac{1}{2}(1 \pm i \sqrt{3})$, the equilateral triangle configurations. Nondegeneracy is checked as above. To show that the function $V(z)-i L / \bar{M}=P / Q$ has simple zeros, we examine the numerator $P$, a cubic polynomial, which simplifies to

$$
-2\left(z_{3}^{2}+2\right)-\left(z_{3}-1\right)\left(z_{3}+1\right)\left(z_{3}-3\right) .
$$

Its derivative is quadratic, so one may find the roots of $P^{\prime}$ and check that they are not roots of $P$. Thus $P$ has three distinct simple roots, as claimed.

\section{Chapter 3. Stationary Configurations AND The Diagonal}

3.1. Introduction. Stationary configurations are the zeros of rational functions with poles on $\Delta$. It is of interest to see how close these zeros can be to $\Delta$. In this chapter it is proved that if $\kappa$ is regular (as defined below) then there is a neighborhood of $\Delta$ in $\mathbf{P}^{n-2}$ containing no stationary configurations. Consequently the set of stationary configurations is a closed subset of a compact set, hence compact. The existence of such a neighborhood is needed to show that the degree of a component of configuration space (see $\S 6.3$ ) is well defined. The compactness shows that the sets of equilibrium configurations and rigidly translating configurations are projective varieties. See Propositions 3.2.3, 3.2.5, and 3.3.3; this also plays a role in Chapter 5.

Definition 3.1.1. $\kappa=\left(\kappa_{1}, \ldots, \kappa_{n}\right) \in(\mathbf{C}-\{0\})^{n}$ is called $e$-regular if, for every proper subset $I$ of $\{1, \ldots, n\}$,

$$
\sum_{l<j} \kappa_{l} \kappa_{j} \neq 0
$$

(Here $\Sigma_{I}$ denotes summation over indices in $I$.)

$\kappa$ is called regular if $\kappa$ is e-regular, and if $\sum_{I} \kappa_{l} \neq 0$ for every $I \subsetneq\{1, \ldots, n\}$.

Note that we allow the $\kappa_{l}$ to take on complex values; this generalization is not needed until Chapter 5. Also note that Lemma 1.2.1 holds for complex $\kappa_{l}$ as well.

Definition 3.1.2. For $z \in \mathbf{C}^{n}$, define

$$
\rho(z)=\frac{1}{n(n-1)} \sum_{l<j}\left|z_{l}-z_{j}\right| .
$$

This is the average intervortex distance. For $I \subsetneq\{1, \ldots, n\}$, define $\rho_{I}(z)$ in the obvious way.

Definition 3.1.3. The set $B_{\varepsilon}$, for $\varepsilon>0$, is given as follows. Let $[z] \in N$, and pick a representative $z \in \mathbf{C}^{n}$ with $\sum\left|z_{l}\right|^{2}=1$. Then $[z] \in B_{\varepsilon}$ if there is $0<\varepsilon^{\prime}<\varepsilon$, and a partition of $\{1, \ldots, n\}$ into equivalence classes $I_{1} \cup \cdots \cup I_{r}, r<n$, so that $\left|z_{l}-z_{j}\right|$ $<\left(\varepsilon^{\prime}\right)^{2}$ if $l \sim j$, and $\left|z_{l}-z_{j}\right|>\varepsilon$ if $l+j$. An equivalence class $I_{j}$ with more than one element will be called a subcollection. 
Note that $B_{\varepsilon}$ is an open subset of $\mathbf{P}^{n-2}$ which contains $\Delta$. The idea of the following lemmas is to show that if $\varepsilon$ is small and $[z] \in B_{\varepsilon}$ is a stationary configuration, then the subcollections of $[z]$ are very nearly equilibria or rigidly translating configurations.

The lemmas depend on a series of estimates, in which constants appear, depending only on $\left|\kappa_{l}\right|$ and $n$, and obvious in context. Rather than spell them out each time, we will simply write $C_{1}, C_{2}$, etc. Examination of the proofs will show they hold if $V_{l}$ is replaced by $\bar{V}_{l}$ throughout. This is used in Proposition 3.3.3.

\subsection{Equilibria and rigidly translating configurations.}

LEMMA 3.2.1. Suppose $\kappa$ is e-regular and $L \neq 0$. Then the function $f=\rho(z) \sum\left|V_{l}\right|$, which is well defined on $N$, is bounded away from 0 on $N$ :

$$
f([z])>b>0 \quad \forall[z] \in N
$$

for some fixed b. Moreover, $(*)$ holds with the same bound $b$ if $\kappa$ is replaced by $\kappa^{\prime}$ sufficiently near $\kappa$ in $\mathbf{C}^{n}$.

Proof. By induction. Clearly it is true for $n=2$; so assume the lemma holds for fewer than $n$ vortices. Now, $\Sigma\left|V_{l}\right|=0$ only if $z$ is an equilibrium. Since $L \neq 0$, there is no such $z$, and $f$ is bounded away from 0 on the compact set $\mathbf{P}^{n-2}-B_{\varepsilon}$ for all $\varepsilon>0$. Now consider some point $[z] \in B_{\varepsilon}$, with normalized representative $z$ satisfying $\Sigma\left|z_{l}\right|^{2}=1$. Let $I$ be a subcollection with $\left|z_{l}-z_{j}\right|<\varepsilon^{2} \forall l, j \in I$. For $l \in I$, define

$$
V_{l}^{\prime}=\sum_{l \neq j} \frac{i \kappa_{j}}{\overline{z_{l}-z_{j}}}
$$

so that $\left|V_{l}-V_{l}^{\prime}\right|<C_{1} / \varepsilon$ for some constant $C_{1}$. Now,

$$
\sum\left|V_{l}\right|>\sum_{I}\left|V_{l}\right| \geqslant \sum_{I}\left|V_{l}^{\prime}\right|-C_{2} / \varepsilon
$$

for some constant $C_{2}$. However, $\rho_{I}(z)<\varepsilon^{2}$, and $\rho_{I}(z) \sum_{I}\left|V_{l}^{\prime}\right|>b>0$ for some constant $b$ by the induction hypothesis. So

$$
\sum\left|V_{l}\right|>b / \varepsilon^{2}-C_{2} / \varepsilon
$$

which tends to $\infty$ as $\varepsilon \rightarrow 0$. So for $\varepsilon$ sufficiently small, $f$ achieves its minimum in $\mathbf{P}^{n-2}-B_{\varepsilon}$, and is bounded away from 0 . Moreover, the inequalities are valid for $\kappa^{\prime}$ if $\sum\left|\kappa_{l}-\kappa_{l}^{\prime}\right|$ is sufficiently small.

Lemma 3.2.2. Suppose $\kappa$ is e-regular. Then for $\varepsilon$ sufficiently small, $B_{\varepsilon}$ contains no equilibrium configurations.

Proof. In Lemma 3.2.1 we proved, independent of $L$, that $\sum\left|V_{l}\right| \neq 0$ in $B_{\varepsilon}$ for $\varepsilon$ sufficiently small.

PROPOSITION 3.2.3. If $\kappa$ is e-regular, then there are a finite number of equilibrium configurations. 
Proof. Let $\Pi_{l}=\Pi_{l \neq j}\left(z_{l}-z_{j}\right)$. Then $P_{l}=\bar{V}_{l} \Pi_{l}$ is a homogeneous polynomial, and the set

$$
V=V\left(P_{1}, \ldots, P_{n}\right):=\left\{[z] \in \mathbf{P}^{n-2} \mid P_{l}([z])=0 \forall l\right\}
$$

is a projective variety containing all equilibrium configurations, plus possibly some points on $\Delta$. We may write $V=V_{d} \cup V_{a}$, where $V_{d}=V \cap \Delta$ and $V_{a}=V-\Delta=V$ $-B_{\varepsilon}$ for $\varepsilon$ sufficiently small. Now $V_{a}$ contains only the equilibrium configurations, and $V_{a}$ is compact. Hence $V_{a}$ is a projective variety (Shafarevich (1977), p. 371). Since $V_{a} \cap \Delta=\varnothing$ and $\Delta$ contains a hyperplane, $V_{a}$ is finite (Shafarevich (1977), p. 50). This concludes the proof.

The number of points in $V_{a}$ is found in Chapter 5 .

We may treat the rigidly translating configurations the same way. Note that $\sum\left|V_{l}-V_{j}\right|=0$ only if $z$ is rigidly translating or an equilibrium.

LEMmA 3.2.4. If $\kappa$ is regular, $L \neq 0$, and $\sigma \neq 0$, then $\rho(z) \sum\left|V_{l}-V_{j}\right|$ is bounded away from 0 on $N$, and the same bound holds for a neighborhood of $\kappa$ in $\mathbf{C}^{n}$. If $\kappa$ is regular then $B_{\varepsilon}$ contains no rigidly translating configurations for $\varepsilon$ sufficiently small.

Proof. Identical to the proofs of Lemmas 3.2.1 and 3.2.2, save for the substitution of $\sum\left|V_{l}-V_{j}\right|$ for $\sum\left|V_{l}\right|$ and regular for $e$-regular.

PROPOSITION 3.2.5. 'If $\kappa$ is regular, then there are a finite number of rigidly translating configurations.

Proof. Let $V=V\left(P_{l} M+i L \Pi_{l}, l=1, \ldots, n\right)$. If $V_{a}=V-\Delta$, then $V_{a}$ is compact, contains all rigidly translating configurations, and is disjoint from a hyperplane. Therefore $V_{a}$ is finite.

3.3. Relative equilibria. Suppose that $V_{l}=i \lambda z_{l} \forall l$, for some $0 \neq \lambda \in \mathbf{R}$. Observe that $f_{l}=\sum_{j}\left|\left(V_{l}-V_{j}\right) / V_{l}\right| \neq 0$ except at equilibria and rigidly translating configurations.

Lemma 3.3.1. Assume $\kappa$ is regular, and that $L$ and $\sigma$ are both nonzero. Let

$$
f_{l}= \begin{cases}\sum_{l \neq j}\left|\frac{V_{l}-V_{j}}{V_{l}}\right|, & \text { if } V_{l} \neq 0, \\ \infty, & \text { if } V_{l}=0 .\end{cases}
$$

Then $f_{l}$ is a continuous $\mathbf{R}^{\infty}$-valued function on $N$, and for every $l, f_{l}$ is bounded away from 0 on $N$. The same bound applies if $\kappa$ is changed to $\kappa^{\prime}$ sufficiently near.

Proof. By induction. The lemma obviously holds for $n=2$, so assume it holds for fewer than $n$ vortices. The $f_{l}$ are continuous functions, even when $V_{l}=0$, for since $\sigma \neq 0$ and $L \neq 0$, at least one numerator must be nonzero. Thus each $f_{l}$ is bounded away from 0 on the compact set $\mathbf{P}^{n-2}-B_{\varepsilon}$.

So let $[z] \in B_{\varepsilon}$, and fix $l$. It will suffice to show that $f_{l}>b$ for some $b>0$ as $\varepsilon \rightarrow 0$. Either $l+j$ for all $j$, or $l \in I$ a subcollection. 
First, suppose the former. Then $\left|V_{l}\right|<C_{1} / \varepsilon$ for some $C_{1}$. There must be a subcollection $I$, and by Lemma 3.2.1 there is $j \in I$ so that $\left|V_{j}^{\prime}\right|>C_{2} / \varepsilon^{2}$, and therefore $\left|V_{j}\right|>C_{2} / \varepsilon^{2}-C_{3} / \varepsilon>C_{4} / \varepsilon^{2}$ for $\varepsilon$ sufficiently small. So

$$
f_{l}>\left|1-V_{j} / V_{l}\right|>\left|1-C_{4} / C_{1} \varepsilon\right|
$$

which goes to $\infty$ as $\varepsilon \rightarrow 0$. So this case is settled.

Next suppose $l \in I$ which contains other elements. According to Lemma 3.2.1, there is $j \in I$ so that $\left|V_{j}^{\prime}\right|>C_{5} / \varepsilon^{2}$, so $\left|V_{j}\right|>C_{5} / \varepsilon^{2}-C_{6} / \varepsilon>2 C_{5} / 3 \varepsilon^{2}$ for $\varepsilon$ sufficiently small. If $\left|V_{l}\right|<C_{5} / 2 \varepsilon^{2}$, then

$$
f_{l}>\left|1-V_{j} / V_{l}\right|>|1-4 / 3|
$$

so $f_{l}$ is bounded away from 0 . If, on the other hand, $\left|V_{l}\right| \geqslant C_{5} / 2 \varepsilon^{2}$,

$$
\left|\frac{V_{l}-V_{j}}{V_{l}}\right| \geqslant\left|\frac{V_{l}-V_{j}^{\prime}}{V_{l}^{\prime}}\right|\left(1+C_{6} \varepsilon\right),
$$

so

$$
f_{l} \geqslant\left[\sum_{I}\left(1-\frac{V_{j}^{\prime}}{V_{l}^{\prime}}\right)\right]\left(1-C_{7} \varepsilon\right) .
$$

The first factor on the right is bounded away from 0 by the induction hypothesis, so $f_{l}$ is also as $\varepsilon \rightarrow 0$. This completes the proof.

LemMA 3.3.2. Suppose $\kappa$ is regular, $L \neq 0$, and $[z]$ satisfies $V_{l}=i \lambda z_{l}$ for all $l$, $0 \neq \lambda \in \mathbf{R}$. Then for $\varepsilon$ sufficiently small, $B_{\varepsilon}$ contains no such $[z]$.

Proof. First assume that $|S|>\varepsilon$, so $|\lambda|<|L| / \varepsilon$. Suppose that $[z] \in B_{\varepsilon}$, and $I$ is a subcollection. Then

$$
\sum_{I}\left|V_{l}\right|=|\lambda| \sum_{I}\left|z_{l}\right|<C_{1} / \varepsilon
$$

for some $C_{1}$. But $\rho_{I}(z)<\varepsilon^{2}$ and $\kappa$ is regular so that $\sum\left|V_{l}^{\prime}\right|>b / \varepsilon^{2}$ for some constant $b \neq 0$, and $\left|V_{l}-V_{l}^{\prime}\right|<C_{2} / \varepsilon$. So

$$
b / \varepsilon^{2}-C_{3} / \varepsilon<\sum_{I}\left|V_{l}\right|<C_{1} / \varepsilon
$$

a contradiction for $\varepsilon$ sufficiently small. So $[z] \notin B_{\varepsilon}$.

Now (for any $S$ ), if $[z] \in B_{\varepsilon}$, we may assume that there is a subcollection $I$, so that $\left|z_{l}\right|>\varepsilon / 2$ for $l \in I$ : otherwise there is only one subcollection, so there must be a $j$ such that $\left|z_{j}\right|>1 / \sqrt{n}$ (since $\sum\left|z_{l}\right|^{2}=1$ ) and $\left|z_{l}-z_{j}\right|>\varepsilon$ for $l \neq j$. Then $\left|V_{j}\right|<C_{4} / \varepsilon$ and $|\lambda|<C_{5} / \varepsilon$, and the previous argument applies.

So assume there is $I$ with $\left|z_{l}\right|>\varepsilon / 2$ for $l \in I$, and thus $\left|V_{l}\right|=\left|\lambda z_{l}\right| \neq 0$. Now for $l, j \in I$,

$$
\left|1-V_{j} / V_{l}\right|=\left|1-z_{j} / z_{l}\right|<2 \varepsilon
$$

so that

$$
f_{l}^{\prime}=\sum_{I}\left|1-\frac{V_{j}}{V_{l}}\right|<C_{6} \varepsilon
$$


for every $l \in I$. However $\left|V_{l}-V_{l}^{\prime}\right|<C_{7} / \varepsilon$ so that

$$
f_{l}^{\prime \prime}=\sum_{I}\left|1-\frac{V_{j}^{\prime}}{V_{l}^{\prime}}\right|<C_{6} \varepsilon+\frac{C_{8} / \varepsilon}{\left|V_{l}^{\prime}\right|} .
$$

By Lemma 3.2.1, we can find $l \in I$ so that $\left|V_{l}\right|>C_{9} / \varepsilon^{2}$, and finally $f_{l}^{\prime \prime}<C_{6} \varepsilon+C_{10} \varepsilon$. By the induction hypothesis, this contradicts Lemma 3.3.1 for $\varepsilon$ sufficiently small.

This proposition will be used in Chapters 6 and 7. We also have an analog of Proposition 3.2.3 for collinear relative equilibria.

PROPOSITION 3.3.3. If $\kappa$ is regular, then the number of collinear relative equilibria is finite.

Proof. First note that Lemma 3.3.2 holds if $V$ is replaced by $\bar{V}$. So for $\varepsilon$ sufficiently small, $B_{\varepsilon}$ contains no common zeros of the functions $F_{l}=\bar{V}_{l}-i \lambda z_{l}$. So if $V=V\left(F_{l} \Pi_{l}, l=1, \ldots, n\right)$ and $V_{a}=V-\Delta$, we find that $V_{a}$ is finite. But $F_{l}(z)=0 \forall l$ if $z$ is a collinear relative equilibrium, so $V_{a}$ contains all such configurations.

This proposition is strengthened in Theorem 6.5.1, which gives a best possible upper bound for the number of points in $V_{a}$.

\section{CHAPTER 4. THE RESTRICTED $n$-VORTEX SYSTEM AND THE IMPLICIT FUNCTION THEOREM}

\subsection{The restricted $n$-vortex system.}

Definition 4.1.1. A restricted n-vortex system is an $n$-vortex system with one circulation, usually $\kappa_{n}$, equal to 0 .

Since a zero vortex does not affect the other vortices, we may remove the zero vortex from a restricted $n$-vortex stationary configuration to find an $(n-1)$-vortex stationary configuration. Conversely, given an $(n-1)$-vortex stationary configuration, we can find a restricted $n$-vortex stationary configuration by properly placing the zero vortex.

LEMMA 4.1.2. If $\left[a_{1}, \ldots, a_{n-1}\right]$ is an $(n-1)$-vortex equilibrium with circulations $\left(\kappa_{1}, \ldots, \kappa_{n-1}\right)$, then $\left[a_{1}, \ldots, a_{n-1}, z_{n}\right]$ is a restricted $n$-vortex equilibrium configuration for exactly $n-2$ values of $z_{n} \in \mathbf{C}$, counted according to multiplicity as zeros of $V_{n}$.

Proof. Clearly $\left[a_{1}, \ldots, a_{n-1}, z_{n}\right]$ satisfies $V_{1}=\cdots=V_{n-1}=0$ for all $z \in \mathbf{C}-$ $\left\{a_{1}, \ldots, a_{n-1}\right\}$. So we must find $z_{n}$ so that

$$
0=i \bar{V}_{n}=\frac{\kappa_{1}}{z_{n}-a_{1}}+\cdots+\frac{\kappa_{n-1}}{z_{n}-a_{n-1}}=\frac{P}{Q},
$$

where $P$ and $Q$ are polynomials in $z_{n}$, and $\operatorname{deg}(P)=n-2$. The zeros of $V_{n}$ are exactly the zeros of $P$ and are distinct from $a_{1}, \ldots, a_{n-1}$. Therefore $V_{n}=0$ for $n-2$ values of $z_{n}$, counted with their multiplicities as zeros of $V_{n}$.

LEMMA 4.1.3. If $\left[a_{1}, \ldots, a_{n-1}\right]$ is an $(n-1)$-vortex rigidly translating configuration then $\left[a_{1}, \ldots, a_{n-1}, z_{n}\right]$ is a restricted n-vortex rigidly translating configuration for exactly $n-1$ values of $z_{n}$, counted according to multiplicity as zeros of $V_{n}-i L / \bar{M}$. 
Proof. Similar to the preceding lemma. Certainly $\left[a_{1}, \ldots, a_{n-1}, z_{n}\right]$ satisfies $V_{1}=\cdots=V_{n-1}=i L / \bar{M}$ for all $z_{n} \in \mathbf{C}-\left\{a_{1}, \ldots, a_{n-1}\right\}$. We need merely solve

$$
0=i \bar{V}_{n}-\frac{L}{M}=\frac{\kappa_{1}}{z_{n}-a_{1}}+\cdots+\frac{\kappa_{n-1}}{z_{n}-a_{n-1}}-\frac{L}{M}=\frac{P}{Q},
$$

where $P$ and $Q$ are polynomials and $\operatorname{deg}(P)=n-1$. The $n-1$ zeros of $P$ are also zeros of $V_{n}-i L / \bar{M}$.

DEFINITION 4.1.4. The (fluid) flow associated to an $n$-vortex system is the function

$$
V(z)=i \sum_{j} \frac{\kappa_{j}}{\overline{z-z_{j}}} .
$$

The modified (fluid) flow is the function $V(z)-i L / \bar{M}$.

In the fluid mechanical system of $n$ vortices, the flow is a time-dependent vector field giving the velocity of the fluid at each point not at a vortex. The modified flow is the flow viewed from a moving frame of reference.

Thus there are $n-2$ distinct restricted $n$-vortex equilibria associated with an $(n-1)$-vortex equilibrium iff the associated flow has only simple zeros, or equivalently, $n-2$ distinct zeros. A similar statement holds for rigidly translating configurations with distinct zeros of the modified flow; there are $n-1$ associated restricted configurations.

LEMMA 4.1.5. If $\left(a_{1}, \ldots, a_{n-1}\right)$ is a nondegenerate $(n-1)$-vortex equilibrium (resp., rigidly translating configuration) with circulations $\left(\kappa_{1}, \ldots, \kappa_{n-1}\right)$, and the associated flow (resp., modified flow) has only simple zeros, then each associated restricted n-vortex equilibrium (resp., rigidly translating configuration) is nondegenerate.

Proof. We prove the statement for equilibria; the other statement is proved similarly. Let $\left(a_{1}, \ldots, a_{n-1}, a_{n}\right)$ be a restricted $n$-vortex equilibrium. It is nondegenerate if

$$
0 \neq\left|\frac{\partial \bar{V}_{l}}{\partial z_{j}}\right|\left(a_{1}, \ldots, a_{n}\right), \quad 3 \leqslant l, j \leqslant n .
$$

Since $\kappa_{n}=0$, we have $\partial \bar{V}_{l} / \partial z_{n}=0$ for all $l<n$. Thus the above determinant is equal to

$$
\left(\frac{\partial \bar{V}_{n}}{\partial z_{n}}\right) \cdot\left|\frac{\partial \bar{V}_{l}}{\partial z_{j}}\right|\left(a_{1}, \ldots, a_{n}\right), \quad 3 \leqslant l, j<n .
$$

The first factor is nonzero if $a_{n}$ is a simple zero of the flow associated with the $(n-1)$-vortex equilibrium $\left(a_{1}, \ldots, a_{n-1}\right)$. The second factor is nonzero iff the $(n-1)$-vortex system is nondegenerate. This proves the lemma.

Now consider collinear relative equilibria. Suppose $\left(\kappa_{1}, \ldots, \kappa_{n-1}\right)$ has $\kappa_{l}>0 \forall l$, and that $\left[a_{1}, \ldots, a_{n-1}\right]$ is a collinear relative equilibrium, satisfying $V_{l}=i \lambda z_{l} \forall l$, where necessarily $\lambda=L / S>0$. Then the flow $V(z)$ is pure imaginary for $z \in \mathbf{R}$. 
The graph of $\operatorname{Im} V(z)$ for $z \in \mathbf{R}$ looks like this:

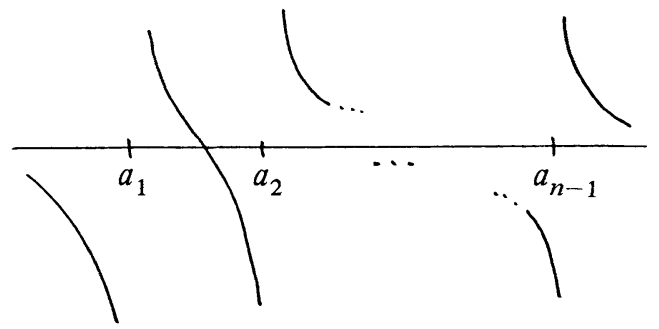

Thus $\operatorname{Im} V(z)$ meets the line $\lambda z$ exactly once in each component of $\mathbf{R}-$ $\left\{a_{1}, \ldots, a_{n-1}\right\}$, a total of $n$ times.

So we have proved the following lemma.

LEMMA 4.1.6. Let $\left[a_{1}, \ldots, a_{n-1}\right]$ be a collinear relative equilibrium of $n-1$ positive vortices. Then $\left[a_{1}, \ldots, a_{n-1}, z_{n}\right]$ is a restricted $n$-vortex collinear relative equilibrium for exactly one value of $z_{n}$ in each component of $\mathbf{R}-\left\{a_{1}, \ldots, a_{n-1}\right\}$.

If we allow not one, but $m$, zero vortices, each $(n-1)$-vortex collinear relative equilibrium is associated with not $n$ but $\left(\begin{array}{l}n \\ m\end{array}\right)$ restricted collinear relative equilibria.

4.2. The implicit function theorem. The link between stationary configurations of the restricted $n$-vortex problem and those of the $n$-vortex system with one weak circulation is the following.

Implicit Function Theorem. Let $f: U \times V \rightarrow \mathbf{R}^{n}$ be a smooth function, where $U \subset \mathbf{R}^{m}, V \subset \mathbf{R}^{n}$ are open sets. Write $f=\left(f_{1}, \ldots, f_{n}\right)$. Assume that $(a, b) \in U \times V$, $f(a, b)=0$, and $\left|\partial f_{l} / \partial x_{j}\right|(a, b) \neq 0$ where the $x_{j}$ are coordinates on $V$. Then there exist open sets $U^{\prime} \subset U, V^{\prime} \subset V$, containing $a$ and $b$ respectively, and a smooth function $g: U^{\prime} \rightarrow V^{\prime}$, having $g(a)=b$, and so that for all $(u, v) \in U^{\prime} \times V^{\prime}, f(u, v)=0 \leftrightarrow$ $g(u)=v$.

Thus $f^{-1}(0)$ is locally a smooth submanifold of $U \times V$ at $(a, b)$, and the projection $(u, v) \mapsto u$ is a coordinate chart on $f^{-1}(0) \cap U^{\prime} \times V^{\prime}$. The theorem remains true if $\mathbf{R}$ is replaced by $\mathbf{C}, x_{j}$ by $z_{j}$, and "smooth" by "complex analytic", throughout.

In outline, the theorem is applied as follows. Let $U$ parametrize the circulations $\kappa_{l}$, and let $V$ be a neighborhood in $\mathbf{C}^{n-2}$ of a restricted $n$-vortex stationary configuration. For the $f_{l}$ we choose the defining functions. Then the function $g$ associates to each choice of circulations a stationary configuration. This method is known as "analytical continuation"; $g$ is said to "continue" the restricted $n$-vortex stationary configuration, away from $\kappa_{n}=0$.

DEFINITION 4.2.1. Let

$$
\begin{aligned}
& K_{L}^{n}=\left\{\left(\kappa_{1}, \ldots, \kappa_{n}\right) \in \mathbf{C}^{n} \mid \kappa_{1}=1 ; \sum \kappa_{l} \kappa_{j}=0\right\}, \\
& K_{\sigma}^{n}=\left\{\left(\kappa_{1}, \ldots, \kappa_{n}\right) \in \mathbf{C}^{n} \mid \kappa_{1}=1 ; \sum \kappa_{l}=0\right\} .
\end{aligned}
$$

Note that $\kappa_{1}=1$ is no restriction, since a change in the circulations by a factor of $\lambda$ simply reparametrizes the time variable $t$ by $1 / \lambda$. 
LEMMA 4.2.2. Suppose $\left[a_{1}, \ldots, a_{n}\right]$ is a nondegenerate equilibrium configuration (resp., rigidly translating configuration). Let $V$ be a neighborhood of $\left[a_{1}, \ldots, a_{n}\right]$ in $N$. Then $\exists$ open $U \subset K_{L}^{n}$ (resp., $K_{\sigma}^{n}$ ) and a complex analytic map $g: U \rightarrow V$ so that, for each $u \in U, V_{1}=\cdots=V_{n}=0$ (resp., $\left.V_{1}=\cdots=V_{n}=i L / \bar{M}\right)$, substituting $\kappa=u$ and $z=g(u)$. In particular, if $u \in \mathbf{R}^{n}$, then $g(u)$ is an equilibrium configuration (resp., rigidly translating configuration). Moreover, each such configuration is nondegenerate.

Proof. We prove the lemma only for equilibria; the other case is similar. Set $f=\left(\bar{V}_{3}, \ldots, \bar{V}_{n}\right)$; this function is analytic in $z_{l}$ and $\bar{\kappa}_{l}$. Since $\left(a_{1}, \ldots, a_{n}\right)$ is nondegenerate,

$$
\left|\frac{\partial f_{l}}{\partial z_{j}}\right|\left(a_{1}, \ldots, a_{n}\right) \neq 0, \quad 3 \leqslant l, j \leqslant n,
$$

by definition. The implicit function theorem applies, and proves the lemma. Nondegeneracy is clear because it is given by the nonvanishing of a continuous function of $u$ which is nonzero at $\left(a_{1}, \ldots, a_{n}\right)$.

Note that by starting with a nondegenerate restricted $n$-vortex equilibrium or rigidly translating configuration, we can continue the circulation of the zero vortex away from zero.

LEMMA 4.2.3. Let $u_{0}=\left(\kappa_{1}, \ldots, \kappa_{n}\right)$ be a choice of circulations which is regular and for which every equilibrium (resp., rigidly translating configuration) is nondegenerate. Let $g_{l}: U_{l} \rightarrow W_{l}, l=1, \ldots, r$ be continuations of these configurations as in Lemma 4.2.2. Then there is an open ball $B_{\varepsilon}\left(u_{0}\right)$ about $u_{0}$ in $K_{L}^{n}$ (resp., $\left.K_{\sigma}^{n}\right)$ such that for any $u \in B_{\varepsilon}\left(u_{0}\right)$, each $z=\left(z_{1}, \ldots, z_{n}\right)$ satisfying $V_{1}=\cdots=V_{n}=0($ resp., $=i L / \bar{M})$ also satisfies $z=g_{l}(u)$ for some $l$. That is, near $u_{0}$ the only equilibria (resp., rigidly translating configurations) are those obtained by continuation from $u_{0}$.

Proof. By Lemmas 3.2.2 and 3.2.4, a neighborhood $B_{\varepsilon}\left(u_{0}\right)$ exists about $u_{0}$ in $K_{L}^{n}$ such that all equilibria are contained in a compact subset $N^{\prime}$ of configuration space $N$. Let $E$ be the compact set $N^{\prime} \times \overline{B_{\varepsilon}\left(u_{0}\right)}$, and $W=W_{1} \cup \cdots \cup W_{r}$. Then $W$ contains all the equilibria over $u_{0}$ and all equilibria obtained by continuation from these. The set $\left\{[z] \mid V_{1}=\cdots=V_{n}=0\right\}$ is closed, hence compact, in $E-W$; and it is disjoint from the closed set $\left(N^{\prime} \times\left\{u_{0}\right\}\right)-W$. Thus the sets have positive distance, and the proof is complete.

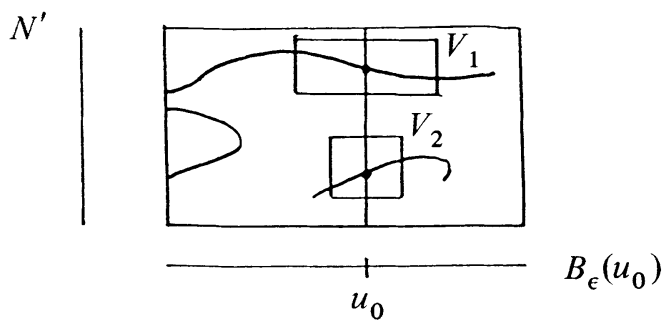


One additional fact about continued solutions is needed.

LEMMA 4.2.4. Let $g: U \rightarrow V$ be a continuation of an equilibrium configuration (resp., rigidly translating configuration) $\left(a_{1}, \ldots, a_{n}\right)$ with circulations $u_{0}=$ $\left(\kappa_{1}, \ldots, \kappa_{n-1}, 0\right)$. Suppose the $(n-1)$-vortex configuration has only simple zeros of the flow (resp., modified flow). Then there exists a deleted neighborhood $U^{\prime}$ of $u_{0}$ such that for $u \in U^{\prime}, g(u)$ also has only simple zeros.

Proof. Postponed until the next section.

We now can apply the implicit function theorem repeatedly, to prove the following fact about the $n$-vortex system.

LEMMA 4.2.5. For every $n \geqslant 3$ there is an open subset of $K_{L}^{n}$ such that for every choice of circulations in it, there are exactly $(n-2)$ ! equilibrium configurations, each of which is nondegenerate and has only simple zeros of the flow.

LEMMA 4.2.6. For every $n \geqslant 3$ there is an open subset of $K_{\sigma}^{n}$ such that for every choice of circulations in it, there are exactly $(n-1)$ ! rigidly translating configurations, each of which is nondegenerate and has only simple zeros of the modified flow.

Proof. We will prove Lemma 4.2.5; the other proof is similar. The claim is true for $n=3$, as was shown in Lemma 2.1. So assume it holds for fewer than $n$ vortices; we need to prove it for $n$.

By the induction hypothesis, there exists a choice of circulations $\left(\kappa_{1}, \ldots, \kappa_{n-1}\right)$ for which there are exactly $(n-3)$ ! nondegenerate equilibria. Each configuration has $n-2$ distinct zeros of the associated flow, so there are a total of $(n-2)$ ! nondegenerate restricted $n$-vortex equilibria. Each may be continued over an open neighborhood of $\left(\kappa_{1}, \ldots, \kappa_{n-1}, 0\right)$, which may be restricted if necessary so that the continued equilibria are the only equilibria (Lemma 4.2.3), are nondegenerate, and have simple zeros of the associated flow (Lemma 4.2.4). This completes the induction, and the proof.

REMARK. In the preceding proof, all but the first three circulations are very small, because they were continued from zero. This restriction is eliminated in the next chapter.

4.3. Bifurcation of zeros. In $\S 3.1$ we saw that each nondegenerate $(n-1)$-vortex equilibrium with distinct zeros of the associated flow gives rise to $n-2$ nondegenerate restricted $n$-vortex equilibria. Each of these can be continued to nondegenerate $n$-vortex equilibria, the associated flow of which having (according to multiplicity) $n-1$ zeros. Since the zero vortex was continued to a weak vortex, the flow does not change much away from a disk around $z_{n}$. We would expect the new zero to be found in this disk. That this is true can be seen in Figures 1 and 2. On a sufficiently small circle around a simple zero of the flow, the flow (as a vector field) has index -1 . Thus there are four points on the circle where the flow is tangent to the circle, and a weak vortex placed at the center of the circle will cancel this flow at two points, causing two zeros of the new flow. 


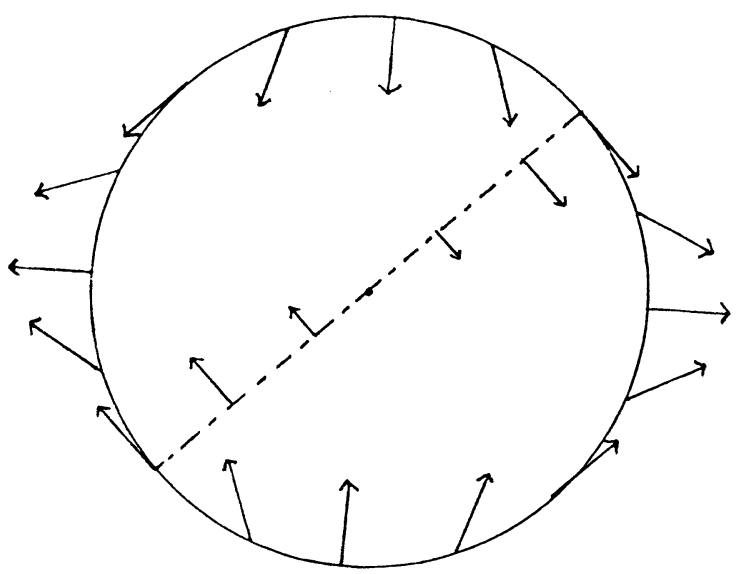

FIGURE 1. Flow near simple zero is $\sim \tilde{z}$.

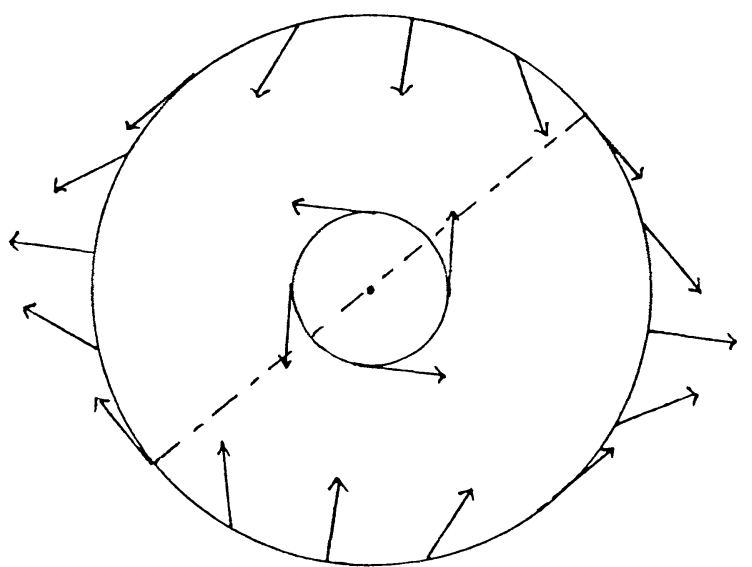

Figure 2. Putting a weak + vortex at 0 results in two zeros on the dotted line.

LEMMA 4.2.4. Let $g: U \rightarrow V$ be a continuation of an equilibrium configuration (resp., rigidly translating configuration) $\left(a_{1}, \ldots, a_{n}\right)$ with circulations $\left(\kappa_{1}, \ldots, \kappa_{n-1}, 0\right)$. Suppose the $(n-1)$-vortex configuration $\left(\kappa_{1}, \ldots, \kappa_{n-1}\right)$ has only simple zeros of the associated flow (resp., modified flow). Then there exists a deleted neighborhood $U^{\prime}$ of $u_{0}$ so that for $u \in U^{\prime}, g(u)$ has only simple zeros of the flow (resp., modified flow).

Proof. We prove the lemma for equilibria; the rigidly translating case is similar. The proof is an application of Rouchés theorem. Let $t$ parametrize a complex line through $u_{0}$. The continuation gives expressions for $z_{1}, \ldots, z_{n}, \kappa_{1}, \ldots, \kappa_{n}$ as functions of $t$. We may assume $\kappa_{n}=t$.

Since $\left(a_{1}, \ldots, a_{n-1}\right)$ is a $(n-1)$-vortex equilibrium when $t=0$, and $z_{n}$ is a simple zero of the flow, we may factor

$$
\begin{aligned}
P_{n} & =i \bar{V}_{n} \Pi_{n}=\left(\frac{\kappa_{1}}{z-z_{1}}+\cdots+\frac{\kappa_{n-1}}{z-z_{n-1}}\right)\left(z-z_{1}\right) \cdots\left(z-z_{n-1}\right) \\
& =h(z)\left(z-z_{n}\right)
\end{aligned}
$$


with $h\left(z_{n}\right) \neq 0$ when $t=0$. The flow of the $n$-vortex system has numerator

$$
\begin{aligned}
P(z) & =\left(\frac{\kappa_{1}}{z-z_{1}}+\cdots+\frac{\kappa_{n-1}}{z-z_{n-1}}+\frac{t}{z-z_{n}}\right)\left(z-z_{1}\right) \cdots\left(z-z_{n}\right) \\
& =h(z)\left(z-z_{n}\right)^{2}+t\left(z-z_{1}\right) \cdots\left(z-z_{n-1}\right) .
\end{aligned}
$$

So $z_{n}$ is a double zero of $P$ when $t=0$, and $P\left(z_{n}\right) \neq 0$ if $t \neq 0$, for $|t|$ sufficiently small.

Consider the rational function $\tilde{P}$ obtained by dividing $P(z)$ by $-\left(z-z_{1}\right) \cdots$ $\left(z-z_{n-1}\right)$ :

$$
\tilde{P}(z)=\tilde{h}(z)\left(z-z_{n}\right)^{2}-t
$$

with $\tilde{h}$ a rational function of $t$ and $z$, and $h\left(z_{n}\right)=1$ when $t=0$ (by reparametrizing $t$ if necessary). For simplicity, let $z_{n}=0$. For fixed $t$, we wish to compare the functions

$$
f_{1}(z)=h(z) z^{2}-t \quad \text { and } \quad f_{2}(z)=z^{2}-t
$$

on the circle $\gamma$ of radius $r=|\sqrt{t}| / 10$ about the point $\sqrt{t}$. The object of the comparison will be to allow the use of Rouché's theorem, which will imply that $f_{2}$ and $f_{1}$ (and hence the $n$-vortex flow) have the same number of zeros within $\gamma$, namely one.

First, $\left|f_{1}-f_{2}\right|=(|h(z)-1|) \cdot|z|^{2}$. On $\gamma,|z|<1.1 \sqrt{t}$. Since $h$ is a rational function of $t,|h(z)-1|$ is bounded by $|t|$ times a constant depending on $|d h / d t|$ evaluated at $z_{n}=0, t=0$. Thus $\left|f_{2}-f_{1}\right|<C_{1} t^{2}$ for some constant $C_{1}$.

Next, $\left|f_{2}\right|=\left|(\sqrt{t}+r \theta)^{2}-t\right|=|t| \cdot\left|\theta / 5+\theta^{2} / 100\right|$ on $\gamma$, where $|\theta|=1$. Thus $\left|f_{2}\right| \geqslant|t| / 10$. Comparing these estimates, $\left|f_{1}-f_{2}\right|<\left|f_{2}\right|$ on $\gamma$ for $|t|$ sufficiently small. Since the zeros of $f_{1}$ are isolated, if $f_{1}$ has a zero on $\gamma$, then the circle may be shrunk slightly without disturbing the inequalities. Rouché's theorem then states that $f_{1}$ and $f_{2}$ have exactly one zero inside $\gamma$. The same argument applies to a similar circle around $-\sqrt{t}$.

Since the location of the other (simple) zeros of the flow are continuous functions of $t$, for $|t|$ sufficiently small, we have proved that the zeros of the flow associated to $g(t)$ are distinct, simple zeros when $t \neq 0$. This holds for every complex line through $u_{0}$, and the lemma is proved.

CHAPTER 5. EQUiLIBRIA AND RIGIDLY TRANSLATING CONFIGURATIONS

5.1. Equilibria of $n$ vortices. It was noted in Chapter 1 that equilibrium configurations for a given choice of circulations $\kappa$ are given by the intersection of a projective variety $V\left(P_{1}, \ldots, P_{n}\right)$ with the open set $\mathbf{P}^{n-2}-\Delta$; the homogeneous polynomials $P_{l}$ are the numerators of the rational functions $\bar{V}_{l}$. If $L=0$ then $V\left(P_{1}, \ldots, P_{n}\right)=$ $V\left(P_{1}, \ldots, P_{n-2}\right)$, so the variety is the common zero set of $n-2$ homogeneous polynomials in $\mathbf{P}^{n-2}$. This is the setting for Bezout's theorem, which says that if $V\left(P_{1}, \ldots, P_{n-2}\right)$ is finite, then it contains exactly $\operatorname{deg}\left(P_{1}\right) \cdots \operatorname{deg}\left(P_{n-2}\right)$ points, counted according to multiplicity. This will be seen for the 4-vortex case in $\$ 8.1$, where two quadratic polynomials are found to have two common zeros on $\Delta$ and two in $\mathbf{P}^{n-2}-\Delta$. These latter points are equilibrium configurations. 
Unfortunately, for $n>4$ the variety $V\left(P_{1}, \ldots, P_{n}\right)$ contains a line in $\Delta$. For example, with $n=5, V\left(P_{1}, P_{2}, P_{3}\right)$ contains the projective line $\left\{z_{1}=z_{2}=z_{3}\right\}$. So Bezout's theorem gives no information about any other zeros that might lie in $\mathbf{P}^{n-2}-\Delta$.

By Lemma 4.2.5, at least for some choices of circulations, there are exactly $(n-2)$ ! nondegenerate equilibrium configurations. The next theorem extends this result to almost every choice of circulations satisfying the necessary condition $\sum \kappa_{l} \kappa_{j}=0$. In this sense it is a converse to the first part of Lemma 1.2.1.

The proof is based on the observation that the $P_{i}$ are homogeneous in $z$ and in the $\bar{\kappa}_{i}$, if they are considered as complex variables. Since only conjugates of the circulations appear, we will replace $\bar{\kappa}_{i}$ by $\kappa_{i}$ throughout; then $V\left(P_{1}, \ldots, P_{n}\right)$ is a variety in $\mathbf{P}^{n-2} \times K_{L}^{n}$. The results of Chapter 3 allow us to separate out the "excess intersection" in $\Delta \times K_{L}^{n}$; then the nature of the fiber of the projection $\mathbf{P}^{n-2} \times K_{L}^{n}$ $\rightarrow K_{L}^{n}$ is examined.

THEOREM 5.1.1. For $n \geqslant 3$, there exists a subvariety $X^{n-1} \subset K_{L}^{n}$ of codimension 1 such that for every choice of circulations $\kappa \in K_{L}^{n}-X^{n-1}$, there exist exactly $(n-2)$ ! equilibrium configurations, which are nondegenerate and have distinct zeros of the flow.

Proof. Let $U \subset K_{L}^{n}$ be the subset of circulations that are $e$-regular. This set is open in both the analytic and Zariski topologies. By Lemma 3.2.2, for each $\kappa \in U$ there is a neighborhood $U_{1}(\kappa) \subset U$ containing $\kappa$ and $U_{2}(\kappa) \subset \mathbf{P}^{n-2}$ containing $\Delta$ so that $U_{2}(\kappa) \times U_{1}(\kappa) \subset \mathbf{P}^{n-2} \times U$ contains no equilibrium configurations. Thus $\Delta \times$ $U$ is contained in an analytic open set disjoint from $V\left(P_{1}, \ldots, P_{n}\right)-\Delta \times U:=V_{a}$. Thus $V_{a}$ is Zariski-closed in $\mathbf{P}^{n-2} \times U$.

The projection $\pi: V_{a} \rightarrow \pi\left(V_{a}\right) \subset U$ onto the second factor takes Zariski closed sets to closed sets (Shafarevich (1977), p. 45). Therefore $\pi\left(V_{a}\right)$ is closed in $U$. By Lemma 4.2.5, $\pi\left(V_{a}\right)$ contains an analytic open set $W$ of dimension $n-2$; since $K_{L}^{n}$ is irreducible, $\pi\left(V_{a}\right)=U$. Since $\operatorname{dim} \pi^{-1}(n)=0$ for all $u \in W, \operatorname{dim} V_{a}=n-2$ and $\pi$ is a map between varieties of the same dimension (Shafarevich (1977), p. 60).

On $V_{a}, J=\left|\partial P_{l} / \partial z_{j}\right|$ is a form, nonzero on $\pi^{-1}(W) \subset V_{a}$. Thus $V_{a}(J)=\{p \in$ $\left.V_{a} \mid J(p)=0\right\}$ has codimension one in $V_{a}$, as does $\pi\left(V_{a}(J)\right) \subset U$. The Zariski open set $U-\pi\left(V_{a}(J)\right)$ is the set of regular values of $\pi$, considered as a smooth map between manifolds of the same dimension. Then the number of points in the inverse image is locally constant on $U-\pi\left(V_{a}(J)\right)$. Since this set is connected, ${ }^{\#} \pi^{-1}(n)=$ $(n-2)$ ! by Lemma 4.2.5.

To complete the proof, consider the form $R=\operatorname{Res}\left(f, f^{\prime}\right)$, where

$$
f=\left(\frac{\kappa_{1}}{z-z_{1}}+\cdots+\frac{\kappa_{n}}{z-z_{n}}\right)\left(z-z_{1}\right) \cdots\left(z-z_{n}\right)
$$

and Res is the resultant. If $R(p) \neq 0$ for some $p \in V_{a}$, then the configuration $p$ has distinct zeros of the flow. Since $R \neq 0$ on $\pi^{-1}(W), \pi\left(V_{a}(R)\right)$ has codimension one in $U$. Setting

$$
X^{n-1}=\left(K_{L}^{n}-U\right) \cup \pi\left(V_{a}(R) \cup V_{a}(J)\right)
$$

completes the proof. 
REMARK. The geometric proof also has an algebraic form. Once $V_{a}$ is established as a variety, Lemma 4.2.5 combines with Proposition 3.17 of Mumford, Algebraic geometry I, p. 46, which is proved over the field of complex numbers, to prove Theorem 5.1.1. Alternatively, $\pi$ is a flat morphism of smooth varieties (since all fibers have the same dimension), and therefore all fibers have the same number of points (cf. Milne, Étale cohomology, Chapter 1).

5.2. Rigidly translating configurations. Since rigidly translating configurations are contained in

$$
V=\left(\left(M \bar{V}_{l}+i L\right) \Pi_{l}, l=1, \ldots, n-2\right) \in \mathbf{P}^{n-2}
$$

we would expect to use Bezout's theorem. Again, however, there is excess intersection in $\Delta$. The same proof as in Theorem 5.1.1, combined with Lemma 4.2.6, gives

THEOREM 5.2.1. For $n \geqslant 3$, there exists a subvariety $Y^{n-1} \subset K_{\sigma}^{n}$ of codimension one such that for every choice of circulations $\kappa \in K_{\sigma}^{n}-Y^{n-1}$, there are exactly $(n-1)$ ! rigidly translating configurations which are nondegenerate and have distinct zeros of the modified flow.

\section{Chapter 6. Collinear Relative eQuilibria With $L \neq 0$}

6.1. Critical points in configuration space. In Chapter 1 it was shown that relative equilibria are exactly the critical points of the modified Hamiltonian

$$
\tilde{H}=-\frac{1}{2} \sum_{l<j} \kappa_{l} \kappa_{j} \ln \frac{\left|z_{l}-z_{j}\right|^{2}}{|S|} .
$$

This function has $|\tilde{H}| \rightarrow \infty$ on a subset of $D=\Delta \cup V(S)$, where $V(S)=\{S=0\}$ $\subset \mathbf{P}^{n-2}$.

If we restrict to the space of collinear configurations, we find that $D$ divides $\mathbf{R} P^{n-2}$ into components. The components of $\mathbf{R} P^{n-2}-\Delta$ can be identified with the equivalence class of the permutation $\sigma$ of $\{1, \ldots, n\}$ which gives the ordering of $\left(z_{1}, \ldots, z_{n}\right)$ along the real line; the equivalence relation is that $\sigma_{1} \sim \sigma_{2}$ if $\sigma_{1}(i)=\sigma_{2}(i)$ or $\sigma_{1}(i)=\sigma_{2}(n+1-i)$ for all $1 \leqslant i \leqslant n$. The components of $\mathbf{R} P^{n-2}-\Delta$ are further divided by the hypersurface $V(S)$; the two components may be distinguished by the sign of $S$.

Suppose that $\kappa_{l}>0$ for all $l$. Then $V(S)=\varnothing$, and $\mathbf{R} P^{n-2}-\Delta$ has $n ! / 2$ components. Moreover, $\tilde{H} \rightarrow \infty$ on the boundary of each component, so $\tilde{H}$ must have a minimum in each component. Direct calculation shows that every critical point of $\tilde{H}$ must be a maximum. We conclude that there are precisely $n ! / 2$ collinear relative equilibria, each nondegenerate (Palmore (1982), p. 717).

6.2. One negative vortex. If one circulation is negative, then $V(S) \neq \varnothing$, and $\tilde{H}$ does not necessarily go to $\infty$ at the diagonal set. To illuminate the general case, consider the following example.

Let $\kappa=(1,1,1,-.6)$ be a choice of circulations for a 4-vortex collinear system. We may identify configuration space with $\mathbf{R} P^{2}$ by choosing for a representative of $\left[z_{1}, z_{2}, z_{3}, z_{4}\right]$ the translate $[x, y, z, w]$ having $w=0$. Then $(x, y, z) \in \mathbf{R}^{3}$; we may normalize by scaling so that $x^{2}+y^{2}+z^{2}=1$. Then $\mathbf{R} P^{2}$ is obtained by identifying 
antipodal points, or equivalently, restricting to the subset $\{z \geqslant 0\}$, and identifying antipodal points on the equator $\{z=0\}$. Figure 3 shows the projection of this set onto the $(x, y)$ plane, as well as the image of the diagonal set $\Delta$. Figure 4 shows $V(S)$.

Now the boundary of the component marked $(*)$ in Figure 4 is made up of four parts. On $V(S)$ and $\{y=0\}, \tilde{H} \rightarrow-\infty$. On the other two, $\tilde{H} \rightarrow \infty$. Therefore, $\tilde{H}$ must have a saddle point in this component. The same is true for the other components of $\mathbf{R} P^{n-2}-D$ in the first quadrant of the $(x, y)$ plane having $S>0$. Thus there are at least six collinear relative equilibria.

We now generalize to the $n$-vortex system.

THEOREM 6.2.1. Suppose $\left(\kappa_{1}, \ldots, \kappa_{n}\right)$ is a choice of $n$ circulations with $\kappa_{n}$ the only negative one, so that $L$ is positive. Let $s$ be the integer so that $\kappa_{l}+\kappa_{n}>0$ holds for exactly s choices of $l<n$. Then there are at least $s(n-2)$ ! collinear relative equilibria.

Proof. There are exactly $s(n-2)$ ! components of $\mathbf{R} P^{n-2}-\Delta$ having order $(n, l, \ldots)$, with $\kappa_{l}+\kappa_{n}>0$. We will show that each such component $C$ contains at least one critical point of $\tilde{H}$.

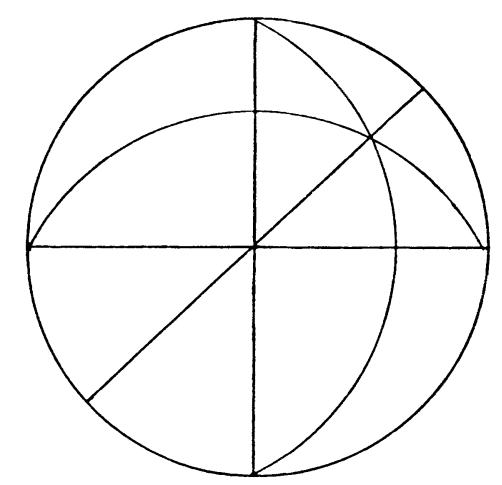

FIGURE 3. Configuration space with diagonals $(n=4)$.

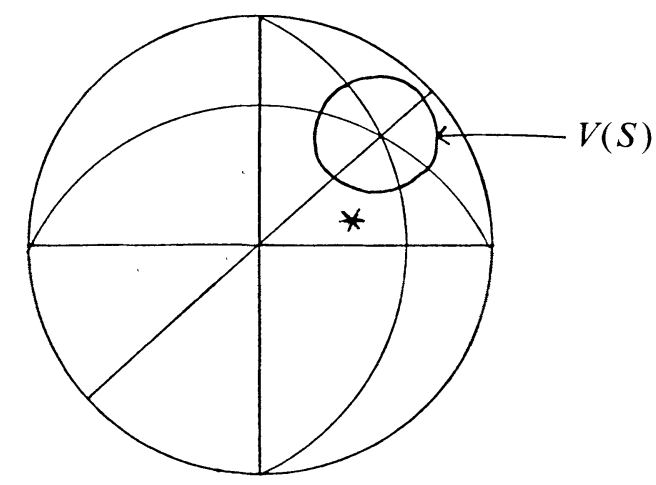

Figure 4. The set $V(S)=\{S=0\}$. 
Since $S$ is negative at the point of $\partial C$ (the boundary of $C$ ) where $z_{1}=z_{2}=\cdots$ $=z_{n-1}$ and $S$ is positive at any point of $\partial C$ where $z_{l}=z_{n}$, the set $C$ is divided into two parts by the hypersurface $V(S)$. Let $C_{+}$be the part having $S$ positive.

Now examine the behavior of $\tilde{H}(p), p \in C_{+}$, as $p$ approaches the boundary $\partial C_{+}$. Since $L>0, \tilde{H} \rightarrow-\infty$ as $p \rightarrow V(S) \cap \partial C_{+}=C_{1}$. Also $\tilde{H} \rightarrow-\infty$ as $p$ nears the subset $C_{2}$ of $\partial C_{+}$where $z_{l}$ and $z_{n}$ coincide. $C_{1}$ and $C_{2}$ are disjoint, since $S$ is bounded away from zero on $C_{2}$.

On the other hand, since $\kappa_{l}>0$ for $l<n, \tilde{H} \rightarrow \infty$ as $p$ nears any point in $\partial C_{+}$ not in $C_{1} \cup C_{2}$.

If $n=4$, it follows that $C$ must contain a saddlepoint, as in the example preceding the theorem. For larger $n, \partial C_{+}-\left(C_{1} \cup C_{2}\right)$ is connected. We conclude that, for $\tilde{H}$ restricted to $C_{+}, \tilde{H}^{-1}((-\infty, a])$ has two components for sufficiently small $a, \tilde{H}^{-1}([b, \infty))$ has one component for $b$ sufficiently large, and $\tilde{H}$ is continuous on the compact set $H^{-1}([a, b])$. So by critical point theory $\tilde{H}$ must contain a critical point in $\tilde{H}^{-1}([a, b]) \subset C_{+}$. This critical point is a collinear relative equilibrium, and the theorem is proved.

6.3. The degree of a component of $\mathbf{R} P^{n-2}-\Delta$. Let $C$ be a component of $\mathbf{R} P^{n-2}-\Delta$, with boundary $\partial C$ and closure $\bar{C}=C \cup \partial C$. Since $\Delta$ contains a hyperplane disjoint from $\partial C$, we may consider an affine neighborhood $\mathbf{R}^{n-2}$ of $C$ in $\mathbf{R} P^{n-2}$, in which $C$ appears as a bounded component of the complement of a finite number of hyperplanes. Thus $C$ is a bounded, convex subset of $\mathbf{R}^{n-2}$, homeomorphic to the open unit ball $B$, and $\partial C$ is homeomorphic to $\partial B=S^{n-3}$.

Suppose $\kappa=\left(\kappa_{1}, \ldots, \kappa_{n}\right)$ is a regular choice of circulations, with $L \neq 0$. According to Lemma 3.3.2, there is an open neighborhood $U$ of $\partial C$ in $\bar{C}$ which contains no zeros of the vector field grad $\tilde{H}$. Pick an interior point $p_{0}$ of $C$ (e.g., the centroid) and let $f_{t}: \bar{C} \times[0,1] \rightarrow \bar{C}$ be the straight-line contraction of $\bar{C}$ to $p_{0} ; f_{0}=$ id and $f_{1} \equiv p_{0}$. For $\varepsilon$ sufficiently small, $f_{\varepsilon}(\partial C) \subset U$ and $f_{\varepsilon}(\partial C)$ can be smoothed in $U$ to a set $N$ diffeomorphic to $S^{n-3}$. The vector field grad $\tilde{H}$ is nonzero on $N$, but undefined on $N \cap\{S=0\}$. However, the unit vector field $V=(\operatorname{grad} \tilde{H}) /\|\operatorname{grad} \tilde{H}\|$ is well defined on all of $N$ : since $L \neq 0, \operatorname{grad} \tilde{H} \rightarrow-L z / S$ near $\{S=0\}$, so that $V$ has a removable singularity there.

Definition 6.3.1. The degree of $C$, deg $C$, is the degree of the map $N \rightarrow S^{n-3}$ given by $V$.

We must check that deg $C$ does not depend on the choice of smoothing $N$. If $N^{\prime}$ is another choice then $N$ is homotopic to $N^{\prime}$ along the contraction because $V$ is defined in $U$; this gives a homotopy between $f: N \rightarrow S^{n-3}$ and $f^{\prime}: N \rightarrow S^{n-3}$. Consequently the degree is the same.

The significance of this degree is that if $\operatorname{deg} C \neq 0$ then $C$ contains a zero of grad $\tilde{H}$, that is, a collinear relative equilibrium configuration.

Definition 6.3.2. $K_{r}^{n}=\left\{\kappa \in \mathbf{R}^{n} \mid \kappa_{1}=1 ; \kappa\right.$ is regular $\}$.

Proposition 6.3.3. Fix some component $C$ of $\mathbf{R} P^{n-2}-\Delta$. The map $K_{r}^{n}-K_{L}^{n} \rightarrow \mathbf{Z}$ taking $\kappa \mapsto \operatorname{deg} C$ is constant on connected components. 
Proof. Let $\kappa, \kappa^{\prime}$ be two regular choices of circulations with $L \neq 0$, contained in the same component of $K_{r}^{n}-K_{L}^{n}$. This component is clearly path-connected, so let $\gamma(t)$ be a smooth path with $\gamma(0)=\kappa, \gamma(1)=\kappa^{\prime}$. The image $\gamma([0,1])$ is compact.

It follows from Lemma 3.3.2 that there is an open set $U \subset \bar{C}$ containing $\Delta$ which contains no zero of grad $\tilde{H}$, for any choice of circulations in $\gamma([0,1])$. Let $N$ be a smoothing of $\partial C$ in $U$, and to each $t \in[0,1]$ associate the integer $\operatorname{deg}_{t} C$. Thus $\gamma$ provides a smooth path in $\mathbf{Z}$ between $\operatorname{deg}_{0} C$ and $\operatorname{deg}_{1} C$, so $\operatorname{deg}_{0} C=\operatorname{deg}_{1} C$.

Hence to compute the degree of a component for some choice of circulations $\kappa$ we may compute the degree for some other $\kappa^{\prime}$ in the same component. If the component adjoins a restricted system (some $\kappa_{l}=0$ ) the implicit function theorem comes into play.

Lemma 6.3.4. Assume $\kappa_{l}>0$ for $l=1, \ldots, p$ and $m \leqslant p+1$. Then there exists $\varepsilon>0$ so that if $0 \neq\left|\kappa_{l}\right|<\varepsilon, l=p+1, \ldots, p+m$, then there are at least $p ! / 2$. $\left(\begin{array}{c}p+1 \\ m\end{array}\right)$ nondegenerate collinear relative equilibria of the $(p+m)$-vortex system with circulations $\left(\kappa_{1}, \ldots, \kappa_{p+m}\right)$. There is exactly one solution per component $C_{\alpha}$ of $\mathbf{R} P^{n-2}$ $-\Delta$ having no adjacent negative vortices, and $\operatorname{deg} C_{\alpha} \neq 0$.

Proof. Begin with a simple extension of Lemma 4.1.6 to the case of $m$, rather than one, zero vortex. Precisely, let $\left(a_{1}, \ldots, a_{p}\right)$ be a $p$-vortex collinear relative equilibrium with circulations $\left(\kappa_{1}, \ldots, \kappa_{p}\right)$. There is exactly one such configuration in each of the $p ! / 2$ components of $\mathbf{R} P^{p-2}-\Delta$. By Lemma 4.1 .6 we may position a zero-vortex at exactly $p+1$ points to produce restricted $(p+1)$-vortex relative equilibria, one point in each component of $\mathbf{R}-\left\{a_{1}, \ldots, a_{n}\right\}$. Given $m$ zero-vortices we find $\left(\begin{array}{c}p+1 \\ m\end{array}\right)$ configurations $\left(a_{1}, \ldots, a_{p}, a_{p+1}, \ldots, a_{p+m}\right)$ which satisfy $V_{l}=i \lambda z_{l} \forall l$, for some $\lambda \in \mathbf{R}$.

Next we apply the implicit function theorem to the map $U \times V \stackrel{f}{\rightarrow} \mathbf{R} P^{p+m-2}$, where $U \subset \mathbf{R}^{p+m-2}$ is a neighborhood in $\mathbf{R} P^{p+m-2}$ of $\left(a_{1}, \ldots, a_{p+m}\right)$, or alternatively, a neighborhood in $\mathbf{R}^{p+m-2}$ of $\left(a_{3}, \ldots, a_{p+m}\right)$; and $V$ is a neighborhood of $0 \in \mathbf{R}^{m}$ which parametrizes the circulations $\kappa_{p+1}, \ldots, \kappa_{p+m}$. For $f=\left(f_{3}, \ldots, f_{p+m}\right)$, we take $f_{l}=\operatorname{Im}\left[V_{l}-i(L / S) z_{l}\right]$. The nondegeneracy condition of the implicit function theorem is satisfied at $\left(a_{1}, \ldots, a_{p+m}\right)$ because $\partial f_{l} / \partial z_{j}=0$ and $\partial f_{j} / \partial z_{j} \neq 0$ at $\left(a_{1}, \ldots, a_{p+m}\right)$ for $j>p, l \neq j$, and $\left(a_{1}, \ldots, a_{p}\right)$ is a nondegenerate collinear relative equilibrium.

The implicit function theorem then implies existence of continuations as claimed in the lemma. For suitably small $\varepsilon$, the continued solutions are the only solutions in their respective components. Finally, if the component $C_{\alpha}$ contians a single nondegenerate collinear relative equilibrium, then $\operatorname{deg} C_{\alpha}= \pm 1$. This concludes the proof.

Lemma 6.3.4 combined with Proposition 6.3.3 give

THEOREM 6.3.5. Suppose $\kappa=\left(\kappa_{1}, \ldots, \kappa_{n}\right)$ lies in $K \subset K_{r}^{n}-K_{L}^{n}, K$ a connected component. If $\bar{K}$ contains a point of the form $\left(\kappa_{1}, \ldots, \kappa_{p}, 0, \ldots, 0\right)$ with $\kappa_{l}>0$ for $1 \leqslant l \leqslant p$, and $p \geqslant n / 2$, then the n-vortex system with choice of circulations $\kappa$ has at least $p ! / 2\left(\begin{array}{c}p+1 \\ n-p\end{array}\right)$ collinear relative equilibrium configurations. 
Proof. By Lemma 6.3.4, the claimed number of collinear relative equilibria continue from $\left(\kappa_{1}, \ldots, \kappa_{p}, 0, \ldots, 0\right)$ into the component $K$. Thus for every $\kappa \in K$ at least this number of components of $\mathbf{R}^{n-2}-\Delta$ have nonzero degree. This proves the theorem.

EXAmple. Consider $\kappa=\left(1, \ldots, 1, \kappa_{n}\right)$. For $\kappa_{n}>0$, there are $n ! / 2$ collinear relative equilibria; by Lemma 6.3 .4 , the same is true for negative $\kappa_{n}$ when $\left|\kappa_{n}\right|$ is sufficiently small. It is easy to check that $\kappa$ is regular for $-1 / 2<\kappa_{n}<0$; therefore $n ! / 2$ is a lower bound for the number of collinear relative equilibria when $\kappa_{n}>-1 / 2$. For $\kappa_{n}>-1$, Theorem 6.2.1 applies, and we find a lower bound of $(n-1)$ ! collinear relative equilibria. The results of this chapter give no information if $\kappa_{n} \leqslant-1$.

6.4. Nondegeneracy of collinear relative equilibria. Let $F_{l}=\left(V_{l} S-i L\right) \Pi_{l}$, and $\tilde{F}_{l}$ be obtained from $F_{l}$ by "forgetting" complex conjugation. The $\tilde{F}_{l}$ are polynomials of degree $n$, and $\tilde{F}_{l}=0 \forall l$ for collinear relative equilibrium configurations.

THEOREM 6.4.1. Let

$$
\begin{aligned}
X_{1}=\left\{\kappa \in K_{r}^{n}-K_{L}^{n} \mid\right. & \text { there exist collinear relative } \\
& \text { equilibria for choice of circulations } \kappa\}
\end{aligned}
$$

and

$$
X_{2}=\left\{\kappa \in X_{1} \mid \text { some collinear relative equilibrium is degenerate }\right\} \subset X_{1} .
$$

Then $X_{2}$ is a subvariety of $X_{1}$ with codimension $\geqslant 1$.

Proof. Let

$$
U=\left\{\kappa \in \mathbf{C}^{n} \mid \kappa_{n}=1 \text {, and } \kappa \text { is regular, with } L \neq 0\right\} .
$$

Define $V \subset \mathbf{P}^{n-2} \times U$ by $V=V\left(\tilde{F}_{l}, l=1, \ldots, n\right)$. As in Theorem 5.1.1 and 5.2.1, $V_{a}=V-(\Delta \times U)$ is a variety, which projects to $U$. If $\pi$ is this projection and $p$ is the restriction to real circulations, $p\left(\pi\left(V_{a}\right)\right)=X_{1}$.

According to $\$ 1.3$, there is a form $J$ on $V_{a}$, not identically 0 , which vanishes on degenerate collinear relative equilibria. Therefore $V_{a}(J)$ has codimension 1 in $V_{a}$, and $X_{2}=p\left(\pi\left(V_{a}(J)\right)\right)$ has codimension at least 1 in $X_{1}$.

6.5. An upper bound for collinear relative equilibria. In this section we find an upper bound for the number of collinear relative equilibrium configurations associated with a regular choice of circulations. This upper bound is achieved when all circulations are positive.

THEOREM 6.5.1. If $\kappa$ is a regular choice of $n$ circulations, with $\sigma \neq 0$, and $L \neq 0$, then there are no more than $n ! / 2$ collinear relative equilibrium configurations.

Proof. Using the notation of the preceding theorem, we have the projection of varieties $\pi: V_{a} \rightarrow U$. As in Theorems 5.1.1 and 5.2.1, ${ }^{\#} \pi^{-1}(u)$ is constant for $u$ belonging to a connected Zariski-open subset of $U$. The theorem is proved by computing this number for a specific $u$. 
We proceed by induction. First consider $n=3$ :

$$
\tilde{F}_{1}=\left[\left(\frac{\kappa_{2}}{z_{1}-z_{2}}+\frac{\kappa_{3}}{z_{1}-z_{3}}\right)\left(\kappa_{1} z_{1}^{2}+\kappa_{2} z_{2}^{2}+\kappa_{3} z_{3}^{2}\right)-L\right]\left(z_{1}-z_{2}\right)\left(z_{1}-z_{3}\right) \text {. }
$$

So $\tilde{F}_{1}$ is a cubic polynomial, homogeneous in $\left(z_{1}, z_{2}, z_{3}\right)$, which vanishes at all collinear relative equilibria. On configuration space $\mathbf{P}^{1}-\Delta, \tilde{F}_{1}$ is a cubic polynomial in one variable. If $\kappa_{l}>0$ for all $l, \tilde{F}_{1}=0$ has as solutions exactly the three collinear relative equilibria. Thus ${ }^{\#} \pi^{-1}(u)=3$ for some choice of $u$.

Now assume that for some choice of positive circulations $\left(\kappa_{1}, \ldots, \kappa_{n}\right), V\left(\tilde{F}_{1}, \ldots, \tilde{F}_{n}\right)$ has exactly $n ! / 2$ points, all collinear. Each is associated with $n+1$ nondegenerate restricted $(n+1)$-vortex collinear relative equilibria. By means of the implicit function theorem we continue these solutions to find a choice of $n+1$ positive circulations for which $\left\{\tilde{F}_{l}=0 ; l=1, \ldots, n+1\right\}$ has exactly $(n+1) ! / 2$ solutions, all collinear. This completes the induction, and the proof.

\section{Chapter 7. Collinear RElative EQUilibria AND COLLAPSE CONFIGURATIONS WITH $L=0$}

7.1. One negative vortex. We know from Chapter 1 that relative equilibria with $L=0$ are exactly the critical points of $H$ restricted to the set $X_{n}=\{S=0\} \subset \mathbf{P}^{n-2}$ $-\Delta$. Consider now the collinear configurations. In $\mathbf{R} P^{n-2}-\Delta, X_{n}$ is a hypersurface of codimension 1 . Since $\Delta$ divides $\mathbf{R} P^{n-2}$ into components, it does the same to $X_{n}$. Each component has a characteristic ordering of $\{1, \ldots, n\}$, but not every ordering is associated with a nonempty component of $X_{n}$.

THEOREM 7.1.1. Suppose $\kappa_{l}>0$ for $l=1, \ldots, n-1, \kappa_{n}<0$, and $L=0$. Then there are at least $s(n-2)$ ! collinear relative equilibrium configurations, where $s$ is the number of pairs $(l, n)$ such that $\kappa_{l}+\kappa_{n}>0$.

Proof. Consider any component $C$ of $X_{n}$ having ordering $(n, l, \ldots)$ where $\kappa_{l}+\kappa_{n}>0$. There are $s(n-2)$ ! such components. Let $C^{\prime}$ be the component of $\mathbf{R} P^{n-2}-\Delta$ with the same ordering, i.e., $C \subset C^{\prime}$. Since $S<0$ at the point $\left(z_{1}=\right.$ $\left.\cdots=z_{n-1}\right)$ of $\partial C^{\prime}$ and $S>0$ at the points where $\left(z_{l}=z_{n}\right)$ in $\partial C^{\prime}$, we see that $C$ is nonempty. It is also easy to see that $z_{l} \neq z_{n}$ for all of $\partial C$, so that $H \rightarrow \infty$ everywhere on $\partial C$. Therefore $C$ must contain a maximum of $H$, which is a collinear relative equilibrium configuration.

7.2. Continuation of relative equilibria. We now prove analogs of Proposition 6.3.3, Lemma 6.3.4, and Theorem 6.3.5. The only difficulty is that the components of $X_{n}$ are not constant as the circulations $\kappa$, are varied.

Suppose that $C$ is a component of $X_{n}$, contained in the component $C^{\prime}$ of $\mathbf{R} P^{n-2}-\Delta$. We may take $C^{\prime}$ to be a convex set in $\mathbf{R}^{n-2}$, bounded by hyperplanes. Since grad $S \neq 0$ on $C^{\prime}, C$ is homeomorphic to an open $(n-3)$-ball, and $\partial C^{\prime}$ to $S^{n-4}$. The function $H$ is defined on $C$, and $\operatorname{grad}\left(\left.H\right|_{C}\right)$ is a vector field on $C$. If $\kappa$ is regular, there is a neighborhood $U$ of $\partial C$ in $\bar{C}$ which contains no zeros this vector field, so that $V=\operatorname{grad}\left(\left.H\right|_{C}\right) /\left\|\operatorname{grad}\left(\left.H\right|_{C}\right)\right\|$ is well defined on $U$. As in $\S 6.3$, we may smooth $\partial C$ within $U$ to $N \approx S^{n+4}$. 
Definition 7.2.1. The degree of $C$ is the degree of the map $N \rightarrow S^{n-4}$ given by $V$.

As before, we find that $\operatorname{deg} C$ is independent of the smoothing chosen. If $\operatorname{deg} C \neq 0$ then $C$ contains a collinear relative equilibrium.

THEOREM 7.2.2. Suppose $\kappa, \kappa^{\prime}$ are two points in $K_{r}^{n} \cap K_{L}^{n}$, and $\gamma$ is a path connecting them; write $\kappa_{t}=\gamma(t), \kappa_{0}=\kappa$ and $\kappa_{1}=\kappa^{\prime}$. Let $\sigma$ be a permutation of $\{1, \ldots, n\}$, and let $C_{t}$ be the component of $X_{n}$ associated with $[\sigma]$. If $C_{t} \neq \varnothing \forall t \in[0,1]$, then $\operatorname{deg} C_{0}=\operatorname{deg} C_{1}$.

Proof. It suffices to show that the degree is locally constant along the path $\gamma$. Fix $\tau \in[0,1]$; for $|t-\tau|<\varepsilon$ we have a neighborhood $U$ of $\partial C_{[\sigma]}$ in $\bar{C}_{[\sigma]}$ which contains no zeros of $\operatorname{grad}\left(\left.H\right|_{S=0}\right)$; and for $\varepsilon$ sufficiently small there is a homotopy along grad $S$ between the hypersurfaces $C_{t}$. Along with the path $\gamma$, this provides a continuous map into $\mathbf{Z}, t \mapsto \operatorname{deg} C_{t}$. This proves the theorem.

THEOREM 7.2.3. Let $\kappa, \kappa^{\prime} \in K_{r}^{n} \cap K_{L}^{n}$, with $\kappa=\left(\kappa_{1}, \ldots, \kappa_{n}\right), \kappa^{\prime}=$ $\left(\kappa_{1}, \ldots, \kappa_{p+1}, 0, \ldots, 0\right) ; \kappa_{l}>0$ for $l \leqslant p, \kappa_{p+1}<0 ; n-1 \leqslant 2 p$. Suppose $\kappa, \kappa^{\prime}$ are joined by a path in $K_{r}^{n} \cap K_{L}^{n}$, so that every nonempty component of $X_{n}$ with circulations $\kappa$ is nonempty all along $\gamma$. Then for choice of circulations $\kappa$, there are at least $s(p-1) !\left(\begin{array}{c}p-p \\ n-1\end{array}\right)$ collinear relative equilibrium configurations, where $s$ is the integer so that $\kappa_{l}+\kappa_{p+1}>0$ for $s$ choices of $l \leqslant p$.

Proof. We show that the minimum number $s(p-1) !\left(n_{n-p}^{p}\right)$ of components have nonzero degree at some point along the path; the result then follows from the preceding theorem.

Consider first the $(p+1)$-vortex system $\left(\kappa_{1}, \ldots, \kappa_{p+1}\right)$. By Theorem 7.1.1, there are at least $s(p-1)$ ! collinear relative equilibria, with the negative vortex at one end. For such a configuration, satisfying $V_{l}=i \lambda z_{l} \forall l$, the graph of the flow $V(z)$ looks like this

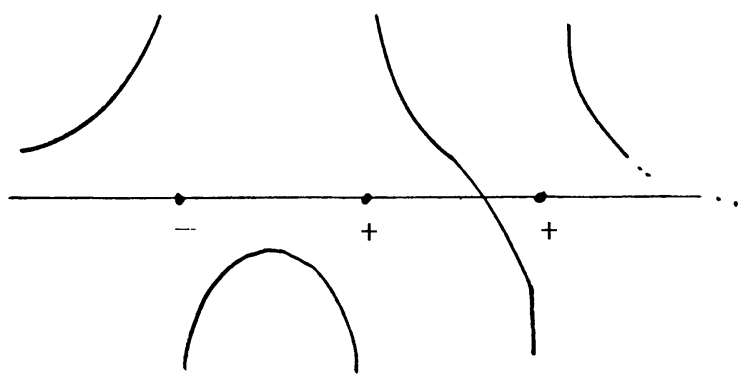

Thus there are at least $p$ solutions to the equation $V(z)=i \lambda z$, and each solution is unique in its component of $\mathbf{R}-\left\{z_{1}, \ldots, z_{p+1}\right\}$. Therefore one obtains $\left({ }_{n-p}^{p} p\right)$ restricted $n$-vortex relative equilibria.

It is proved in the next section (Theorem 7.3.1) that the set of circulations $\left(\kappa_{1}, \ldots, \kappa_{p+1}\right)$ for which these restricted relative equilibria are nondegenerate (that is, satisfy the nondegeneracy condition of the implicit function theorem) is open and dense in $K_{r}^{n} \cap K_{L}^{n}$. We may assume then that they are nondegenerate-otherwise we perturb $\kappa^{\prime}$ slightly, and move the path from $\kappa$ to $\kappa^{\prime}$ accordingly. 
We may therefore continue these restricted solutions slightly along the path to $\kappa$, so that the continued solutions are unique in their components of $X_{n}$. The degrees of these components are nonzero, and the theorem is proved.

REMARK. The $L=0$ circulation set $K_{L}^{n}$ is in no sense "near" the positive-circulation set, so that relative equilibria cannot be continued from relative equilibria with all positive circulations. Therefore Theorem 7.1.1 is essential in providing stationary configurations which can be continued.

7.3. Nondegeneracy of collinear relative equilibria. As in Theorem 6.4.1, let $\kappa=\left(\kappa_{1}, \ldots, \kappa_{n}\right)$ and

$$
F_{l}=V_{l} z_{n}-V_{n} z_{l}, \quad l=1, \ldots, n-3, \quad F_{n-2}=S .
$$

Then denote by $\tilde{F}_{l}$, the rational function of $z$ and $\kappa$ given by "forgetting" conjugation in $F_{l}$. Then $F_{l}=\tilde{F}_{l}=0 \forall l$ at collinear relative equilibria.

THEOREM 7.3.1. Let

$Y_{1}=\left\{\kappa \in K_{r}^{n} \cap K_{L}^{n} \mid\right.$ there exist collinear relative equilibria, with circulations $\left.\kappa\right\}$,

$Y_{2}=\left\{\kappa \in Y_{1} \mid\right.$ some collinear relative equilibrium is degenerate $\}$.

Then $Y_{2} \subset Y_{1}$ is a subvariety of codimension $\geqslant 1$.

Proof. As in Theorem 6.4.1,

$$
V_{a}=V\left(\tilde{F}_{l}\right)-(\Delta \times U) \subset \mathbf{P}^{n-2} \times U
$$

is a variety, where

$$
U=\left\{\kappa \in \mathbf{C}^{n} \mid \kappa_{1}=1, \kappa \text { is regular, } L=0\right\} .
$$

Moreover $V_{a}$ contains all collinear relative equilibria.

By $\S 1.3$, there are forms on $V_{a}$, not identically zero, which vanish at $p \in V_{a}$ only if $\pi(p) \in Y_{2}, \pi$ the projection onto the last factor. Thus $Y_{2}$ has codimension $\geqslant 1$ in $Y_{1}$, as claimed.

7.4. Collapse configurations. In $\S 1.2$ it was conjectured that when $L=0$ the set of collapse configurations has dimension one. This can be put on a firm basis by the following.

THEOREM 7.4.1. Let $n>3$. For each $\kappa \in K_{L}^{n}$, except for a subvariety of codimension 1 , every collinear relative equilibrium solution lies on a one-dimensional family of collapse configurations. Each family is a submanifold except at a finite number of points.

Proof. Let $F_{l}=V_{l} z_{n}-V_{n} z_{l}, 3 \leqslant l \leqslant n-1$, and $F_{n}=\sum \kappa_{l} z_{l}^{2}$. By taking the real and imaginary parts, we obtain $2 n-4$ real functions. As in the preceding theorem, it follows that for each $\kappa \in K_{L}^{n}$ (except for a subvariety of codimension one) the corresponding Jacobian determinant is nonzero at every collinear relative equilibrium. Thus the gradients are independent and we may find a tangent vector $v=\sum\left(a_{j}\left(\partial / \partial x_{j}\right)+b_{j}\left(\partial / \partial y_{j}\right)\right)$ satisfying $D F_{l}(v)=0,3 \leqslant l \leqslant n-1 ; D\left(\operatorname{Re} F_{n}\right)(v)$ $=0, D\left(\operatorname{Im} F_{n}\right)(v) \neq 0$. It is easy to check that some $b_{j} \neq 0$, and that $D F_{l}\left(v^{\prime}\right)=0$ $\forall l<n$ where $v^{\prime}=\sum b_{j}\left(\partial / \partial y_{j}\right)$, that is, $v^{\prime}$ is the "imaginary part" of $v$. It follows 
that $v^{\prime}$ is tangent to the defining functions $\left\{F_{3}, \ldots, F_{n-1}, S\right\}$ of collapse configurations, and the first statement of the theorem follows by the implicit function theorem.

If $X$ is the collapse family, we see that $X$ is a 1-dimensional algebraic variety. The singular points of $X$ form a subvariety of codimension $\geqslant 1$, so that $X$ is smooth at all but a finite number of points. The second statement of the theorem follows, and the theorem is proved.

REMARK. If $n=3$ then the set of collapsing configurations is precisely the circle where $S$ vanishes.

\section{CHAPTER 8. THE 4-VORTEX SYSTEM}

8.1. The 4-vortex equilibria. In Chapter 2 the 3 -vortex equilibrium equation was solved explicitly. The 4-vortex case can be solved similarly, except that there are two quadratics rather than a single linear equation.

THEOREM 8.1.1. There are exactly two solutions to the 4-vortex equilibrium problem when $L=0:\left[z_{1}, \ldots, z_{4}\right]$ with

$$
z_{1}=\frac{2 \kappa_{4}+\kappa_{2} \pm \kappa_{2} \sqrt{-3}}{2\left(\kappa_{2}+\kappa_{3}+\kappa_{4}\right)}, \quad z_{2}=\frac{-\kappa_{2} z_{1}}{\kappa_{1}+\kappa_{3} z_{1}}, \quad z_{3}=1, \quad z_{4}=0 .
$$

Proof. By Proposition 1.1, we must solve the system $\left\{\bar{V}_{3}=0, \bar{V}_{4}=0\right\}$. Since the equations are invariant under translation, set $z_{4}=0$. We seek solutions $\left[z_{1}, z_{2}, z_{3}\right]$ in $\mathbf{P}^{2}$ to

$$
0=\frac{\kappa_{1}}{z_{1}}+\frac{\kappa_{2}}{z_{2}}+\frac{\kappa_{4}}{z_{3}}, \quad 0=\frac{\kappa_{1}}{z_{3}-z_{1}}+\frac{\kappa_{2}}{z_{3}-z_{2}}+\frac{\kappa_{4}}{z_{3}} .
$$

Clearing the denominators gives the homogeneous polynomials

$$
\begin{aligned}
& 0=\kappa_{1} z_{2} z_{3}+\kappa_{2} z_{1} z_{3}+\kappa_{3} z_{1} z_{2} \\
& 0=\kappa_{1} z_{3}\left(z_{3}-z_{2}\right)+\kappa_{2} z_{3}\left(z_{3}-z_{1}\right)+\kappa_{4}\left(z_{3}-z_{1}\right)\left(z_{3}-z_{2}\right) .
\end{aligned}
$$

Solutions to $(*)$ will satisfy the original system unless $z_{1}, z_{2}, z_{3}, z_{1}-z_{2}$, or $z_{2}-z_{3}$ vanishes.

First find the solutions at infinity by setting $z_{3}=0:(*)$ reduces to $0=z_{1} z_{2}$. So there are two such solutions, $[1,0,0]$ and $[0,1,0]$. These are not solutions to the original problem, since these points lie on the diagonal.

Next, if $z_{3} \neq 0$ we may set it to 1 . We find that $(*)$ becomes

$$
0=\kappa_{1} z_{2}+\kappa_{2} z_{1}+\kappa_{3} z_{1} z_{2}, \quad 0=\kappa_{1} y_{2}+\kappa_{2} y_{1}+\kappa_{4} y_{1} y_{2},
$$

where $y_{i}=1-z_{i}$. Rearranging,

$$
z_{2}\left(\kappa_{1}+\kappa_{3} z_{1}\right)+\kappa_{2} z_{1}=0, \quad y_{2}\left(\kappa_{1}+\kappa_{4} y_{1}\right)+\kappa_{2} y_{1}=0 .
$$

Multiply the top equation by $\left(\kappa_{1}+\kappa_{4} y_{1}\right)$, the bottom by $\left(\kappa_{1}+\kappa_{3} z_{1}\right)$, and add; use the fact that $z_{i}+y_{i}=1$,

$$
\left(\kappa_{1}+\kappa_{4} y_{1}\right)\left(\kappa_{1}+\kappa_{3} z_{1}\right)+\kappa_{2}\left[z_{1}\left(\kappa_{1}+\kappa_{4} y_{1}\right)+y_{1}\left(\kappa_{1}+\kappa_{3} z_{1}\right)\right]=0 .
$$

This is quadratic in $z_{1}$,

$$
\begin{aligned}
z_{1}^{2}\left(-\kappa_{3} \kappa_{4}-\right. & \left.\kappa_{2} \kappa_{4}-\kappa_{2} \kappa_{3}\right) \\
& +z_{1}\left(-\kappa_{1} \kappa_{4}+\kappa_{1} \kappa_{3}+\kappa_{3} \kappa_{4}+\kappa_{1} \kappa_{2}+\kappa_{2} \kappa_{4}+\kappa_{2} \kappa_{3}-\kappa_{1} \kappa_{2}\right) \\
& +\left(\kappa_{1}^{2}+\kappa_{1} \kappa_{4}+\kappa_{1} \kappa_{2}\right)=0 .
\end{aligned}
$$


Since $L=0$, this reduces to

$$
z_{1}^{2}\left(\kappa_{2}+\kappa_{3}+\kappa_{4}\right)+z_{1}\left(-2 \kappa_{4}-\kappa_{2}\right)+\left(\kappa_{1}+\kappa_{2}+\kappa_{4}\right)=0 .
$$

The discriminant $D$ reduces to $-3 \kappa_{2}^{2}$. So the solutions follow from the quadratic formula.

Note that in the proof we could as easily have solved for $z_{2}$,

$$
z_{2}=\left(2 \kappa_{4}+\kappa_{1} \mp \kappa_{1} \sqrt{-3}\right) / 2\left(\kappa_{1}+\kappa_{3}+\kappa_{4}\right) .
$$

It is easy to check that $z_{1}$ and $z_{2}$ lie either on the line of slope

$$
\sqrt{3}\left(\kappa_{3}+\kappa_{4}\right) /\left(\kappa_{3}-\kappa_{4}\right)
$$

through the point $(1+i \sqrt{3}) / 2$ or on the conjugate of this line. If $\kappa_{3}=\kappa_{4}$, then $\operatorname{Re} z_{1}=\operatorname{Re} z_{2}=1 / 2$. The formulas indicate that if $\kappa_{1} \rightarrow \infty$, then $z_{1} \rightarrow \infty$ and $z_{2} \rightarrow(1+i \sqrt{3}) / 2$, so that $z_{2}, z_{3}, z_{4}$ form an equilateral triangle with total circulation $\rightarrow 0$. Thus the 3 -vortex rigidly translating configurations can be interpreted as a limiting case of 4-vortex equilibria, with one circulation very large.

Note also that if one circulation is set to zero, the formulas give two solutions to the reduced 4-vortex equilibrium problem. Thus the 3-vortex equilibria have simple zeros of the flow field, as was claimed in Chapter 2.

8.2. Four-vortex rigidly translating configurations. When the total circulation is zero, to find rigidly translating configurations, it suffices to solve the two equations $V_{1}=V_{2}=i L / \bar{M}$. If $z=\left(0,1, z_{3}, z_{4}\right)$ then these read

$$
-\kappa_{2}-\kappa_{3} / z_{3}-\kappa_{4} / z_{4}=\kappa_{1}+\kappa_{3} / y_{3}+\kappa_{4} / y_{4}=L / M,
$$

where $y_{i}=1-z_{i}$. Clearing the denominators,

$$
\begin{aligned}
& 0=L z_{3} z_{4}+\left(\kappa_{2}+\kappa_{3} z_{3}+\kappa_{4} z_{4}\right)\left(\kappa_{2} z_{3} z_{4}+\kappa_{3} z_{4}+\kappa_{4} z_{3}\right), \\
& 0=L y_{3} y_{4}+\left(\kappa_{2}+\kappa_{3} z_{3}+\kappa_{4} z_{4}\right)\left(\kappa_{1} y_{3} y_{4}+\kappa_{3} y_{4}+\kappa_{4} y_{3}\right) .
\end{aligned}
$$

Each cubic equation is only quadratic in each variable, so we may solve each equation for $z_{3}$, using the quadratic formula. Equating these gives a single equation in terms of the single variable $z_{4}$. This can be solved numerically (by Newton's method, for instance) to find rigidly translating configurations for any specific choice of circulations.

If we choose $\kappa=(1,-1, \alpha,-\alpha)$ and $z=(-1,1, x,-x)$, the symmetry simplifies the equations: independently of $x, V_{1}=V_{2}$ and $V_{3}=V_{4}$ hold. We need only solve $V_{1}=V_{3}$ :

$$
\frac{1}{x+1}-\frac{1}{x-1}-\frac{\alpha}{2 x}=\frac{1}{2}-\frac{\alpha}{1+x}-\frac{\alpha}{x-1}
$$

which reduces to $0=4 x(\alpha x-1)-(\alpha+x)\left(x^{2}-1\right)$, a single cubic equation. So long as $|\alpha| \neq 1$, there is at least one real root, corresponding to a collinear rigidly translating configuration. Again, this single equation can be easily solved numerically.

8.3. Vortex angular momentum 0 . It was seen in Chapter 1 that when $L=0$, relative equilibria and collapsing configurations lie on the hypersurface $\{S=0\}$. We would like to describe this hypersurface. 
Let $\left(z_{1}, \ldots, z_{n}\right) \in \mathbf{C}^{n}$ and suppose $\kappa_{l}>0$ for $l=1, \ldots, j$ and $\kappa_{l}<0$ for $l=j+$ $1, \ldots, n$. We may assume $\sigma \neq 0$ (since $L=0$ ), and that $\sigma>0$ (otherwise multiply the circulations by -1$)$. Define

$$
\begin{aligned}
c_{l}=\sqrt{\left|K_{l}\right|}, & c_{+}=\left(c_{1}, \ldots, c_{j}\right) \in \mathbf{R}^{j}, \\
c_{-} & =\left(c_{j+1}, \ldots, c_{n}\right) \in \mathbf{R}^{n-j}, \\
u_{l}=c_{l} z_{l}, & u_{+}=\left(u_{1}, \ldots, u_{j}\right) \in \mathbf{C}^{j}, \\
u_{-} & =\left(u_{j+1}, \ldots, u_{j}\right) \in \mathbf{C}^{n-j}, \\
\sigma_{+} & =\sum_{1}^{j} \kappa_{l}, \quad \sigma_{-}=\sum_{j+1}^{n}\left(-\kappa_{l}\right) .
\end{aligned}
$$

(Note that $\sigma=\sigma_{+}-\sigma_{-}$and $\left\|c_{ \pm}\right\|^{2}=\sigma_{ \pm}$.) The equations $S=0, M=0$ can then be written

$$
\left\|u_{+}\right\|^{2}=\left\|u_{-}\right\|^{2}, \quad c_{+} \cdot u_{+}=c_{-} \cdot u_{-}
$$

where the "dot product" is simply $\sum c_{l} u_{l}$ and is complex-valued. We may write the decomposition $u_{ \pm}=\alpha_{ \pm} c_{ \pm}+v_{ \pm}$, where $\alpha_{ \pm} \in \mathbf{C}$, and $v_{ \pm} \cdot c_{ \pm}=0$. Note that $v_{+}$lies in a $(j-1)$-dimensional subspace and $v_{-}$in a $(n-j-1)$-dimensional subspace.

It is easily verified that

$$
\left\|u_{ \pm}\right\|^{2}=\left|\alpha_{ \pm}\right|^{2}\left\|c_{ \pm}\right\|^{2}+\left\|v_{ \pm}\right\|^{2}=\left|\alpha_{ \pm}\right|^{2} \sigma_{ \pm}+\left\|v_{ \pm}\right\|^{2}
$$

and the equation $M=0$ becomes $\alpha_{+} \sigma_{+}=\alpha_{-} \sigma_{-}$. The relation $S=0$ is now of the form

$$
\left|\alpha_{+}\right|^{2} \sigma_{+}+\left\|v_{+}\right\|^{2}=\left|\alpha_{-}\right|^{2} \sigma_{-}+\left\|v_{-}\right\|^{2}
$$

Using $M=0$, we obtain

$$
\left\|v_{+}\right\|^{2}=\left|\alpha_{-}\right|^{2} \sigma \sigma_{-} / \sigma_{+}+\left\|v_{-}\right\|^{2} .
$$

Proposition 8.3.1. Assume the $n$-vortex system has circulations $\left(\kappa_{1}, \ldots, \kappa_{n}\right)$, with $\sigma>0$ and $\kappa_{l}>0$ for $l \leqslant j$. Let

$$
X_{n}=\left\{\left[z_{1}, \ldots, z_{n}\right] \in \mathbf{P}^{n-2} \mid M=0 ; S=0\right\} .
$$

Then: (a) $X_{2}=\varnothing$,

(b) $X_{3} \approx S^{1}$ if $j=2 ; X_{3}=\varnothing$ otherwise,

(c) $X_{4} \approx S^{3}$ if $j=2$ or $3 ; X_{4}=\varnothing$ otherwise.

Proof. (a) is obvious. If $n=3$, then in the case $j=1$ we find that $v_{+}=0$ and (1) implies that $X_{3}=\varnothing$. If $j=3$, the right-hand side of (1) is zero, and $X_{3}=\varnothing$. Taking $j=2$, however, means that $v_{-}=0$ and so the nonzero solutions of (1) have $\alpha_{-} \neq 0$. Therefore the set of solutions in $\mathbf{P}^{1}$ to (1) are equivalent to the solutions to $\left\|v_{+}\right\|^{2}=1$ with $v_{+} \in \mathbf{C}$, as claimed in (b).

Now take $n=4$. As before we find $X_{4}=\varnothing$ if $j=1$ or 4 . Taking $j=3$, we have $v_{-}=0$ and (1) is (up to equivalence class in $\mathbf{P}^{2}$ ) $\left\|v_{+}\right\|^{2}=1$, with $v_{+} \in \mathbf{C}^{2}$. Therefore $X_{4}$ is homeomorphic to $S^{3}$. Similarly, if $j=2$, the nontrivial solutions of (1) have 
$v_{+} \neq 0$ and in $\mathbf{P}^{2}(1)$ is equivalent to

$$
1=\left|\alpha_{-}\right| \sigma \sigma_{-} / \sigma_{+}+\left\|v_{-}\right\|^{2}
$$

with $\alpha \in \mathbf{C}$ and $v_{-} \in \mathbf{C}$. It follows that $X_{4} \approx S^{3}$, and the proposition is proved.

REMARK. For $n=3$, the conditions $\sigma>0, j=2$ are equivalent to $\kappa_{1} \kappa_{2} \kappa_{3} \sigma<0$. Also it is easy to check that in the 4-vortex system, $L=0$ implies that $j=2$ or 3 . Thus $X_{4}$ is always homeomorphic to $S^{3}$ when $L=0$.

If we restrict to collinear configurations, the above proposition holds, provided that $S^{3}$ is replaced by $S^{1}$, and $S^{1}$ by $S^{0}$.

Definition 8.3.2. $\Delta_{l j}=\left\{[z] \in \mathbf{P}^{n-2} \mid z_{l}=z_{j}\right\}$.

Obviously $\Delta=\bigcup \Delta_{l j}$. Now, the set $X_{n} \cap \Delta_{l j}$ can be identified with the set $X_{n-1}$ where the choice of circulations of the $(n-1)$-vortex system is taken to be $\left(\kappa_{1}, \ldots, \hat{\kappa}_{l}, \hat{\kappa}_{j},\left(\kappa_{l}+\kappa_{j}\right), \ldots, \kappa_{n}\right)$ (the circumflex denotes omission). The identification is the obvious one-put $z_{l}=z_{j}$ in the equations $M=0, S=0$. Therefore, by Proposition 8.3.1, $X_{4} \cap \Delta_{l j}$ is either empty or homeomorphic to $S^{1}$; in fact it is $S^{1}$ iff $j=2$ for the 3-vortex system. For example, $X_{4} \cap \Delta_{12} \approx S^{1}$ iff $\left(\kappa_{1}+\kappa_{2}\right) \kappa_{3} \kappa_{4} \sigma<$ 0 . Furthermore, since $X_{2}=\varnothing$, we see that no two diagonals $\Delta_{l j}, \Delta_{l^{\prime} j^{\prime}}$ intersect in $X_{4}$, provided that $\kappa_{l}+\kappa_{j}$ and $\kappa_{l^{\prime}}+\kappa_{j^{\prime}}$ are nonzero. If one such pair vanishes, then $\Delta_{l j} \cap \Delta_{l^{\prime} j^{\prime}}$ is a single point.

If we restrict attention to collinear 4-vortex configurations with vortex angular momentum 0 , we know that the relative equilibria are exactly the critical points of $H$ restricted to $X_{4}$. If no two diagonals $\Delta_{l j}, \Delta_{l^{\prime} j^{\prime}}$ meet in $X_{4}$, then $H$ is a continuous $\mathbf{R}^{\infty}$-valued function on $X_{4}$, and $|H|=\infty$ only on $\Delta$. Since the Euler characteristic of

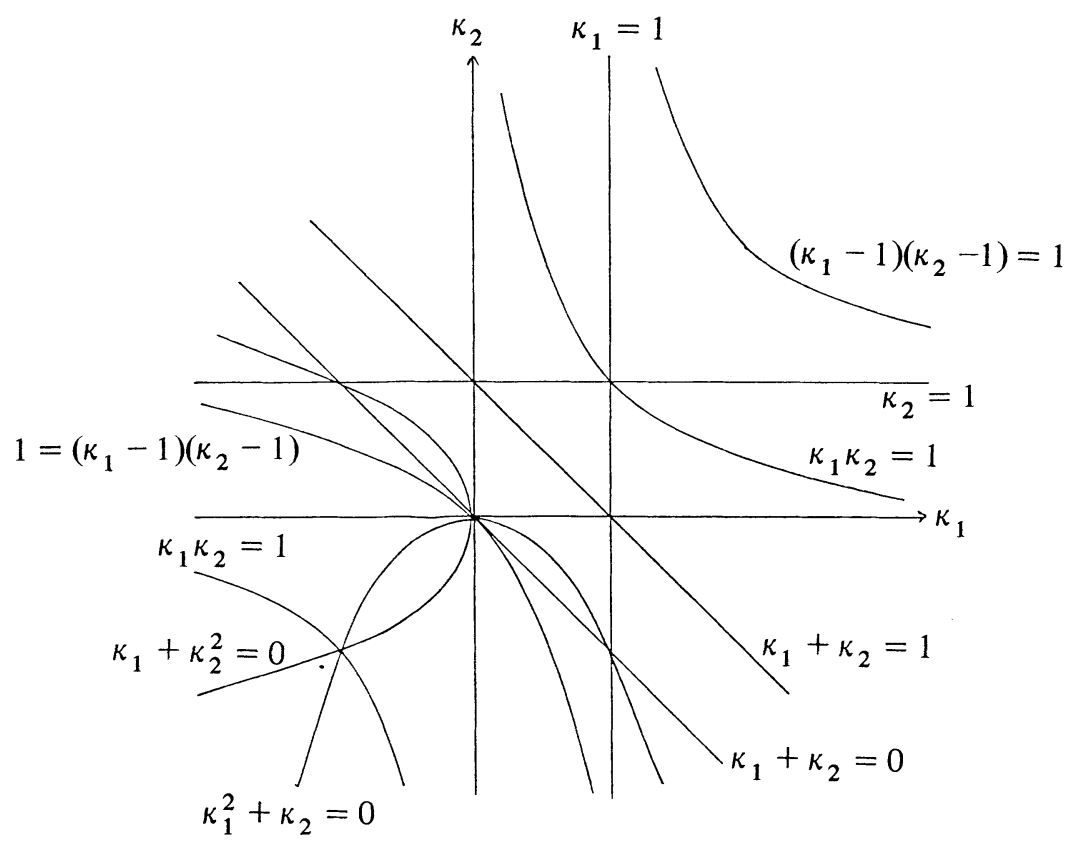

FIGURE 5. The collection of curves $\Gamma$. 


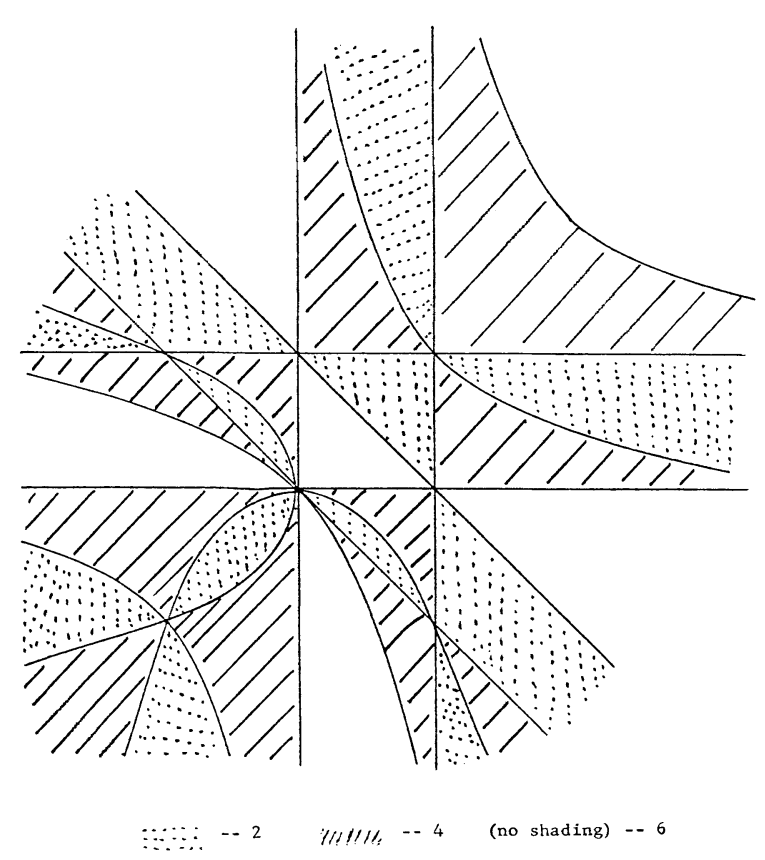

FIGURE 6. The lower bound for the number of collinear relative equilibria.

$X_{4}$ is 1 , any smooth function on $X_{4}$ must have as many maxima as minima. Thus we have the following.

THEOREM 8.3.3. Let $\left(\kappa_{1}, \kappa_{2}, \kappa_{3}, \kappa_{4}\right)$ be a regular choice of circulations with $L=0$. Let $s(p)$ be the sign of $H$ at $p \in X_{4} \cap \Delta$, that is, $s(p)= \pm 1$ if $H(p)= \pm \infty$, respectively. Then there are at least $\left|\sum_{p \in X_{4} \cap \Delta} s(p)\right|$ collinear relative equilibrium configurations.

To compute this lower bound, note that the sign of $H$ at a point of $\Delta_{l j} \cap X_{4}$ is the sign of $\kappa_{l} \kappa_{j}$. The contribution of $\Delta_{l j} \cap X_{4}$ depends on the number of points in it and the sign of $\kappa_{l} \kappa_{j}$.

If we choose $\left(\kappa_{1}, \kappa_{2}\right) \in \mathbf{R}^{2}, \kappa_{4}=-1$, and pick $\kappa_{3}$ so that $\sum \kappa_{l} \kappa_{j}=0$, then it is easy to check that the set of all regular $\left(\kappa_{1}, \kappa_{2}, \kappa_{3}, \kappa_{4}\right)$ correspond to the complement in $\mathbf{R}^{2}$ of the union $\Gamma$ of these curves

$$
\begin{array}{ll}
\kappa_{1}=0, & \kappa_{1}+\kappa_{2}-1=0, \\
\kappa_{2}=0, & \kappa_{1}^{2}+\kappa_{2}=0, \\
\kappa_{1}-1=0, & \kappa_{1}+\kappa_{2}^{2}=0, \\
\kappa_{2}-1=0, & \left(\kappa_{1}-1\right)\left(\kappa_{2}-1\right)=0, \\
\kappa_{1} \kappa_{2}-1=0, & \\
\kappa_{1}+\kappa_{2}=0 . &
\end{array}
$$

Figure 5 shows the union of curves $\Gamma$, and Figure 6 shows the value of the lower bound of Theorem 8.3.3 for each of components of $\mathbf{R}^{2}-\Gamma$. 
From these diagrams it is seen that there exist collinear relative equilibria for almost every choice of circulations in $K_{L}^{4}$. However, it appears that there is at least one choice of circulations, $(1,1,1,-1)$, for which there are no collinear equilibria. By using the parametrization of $X_{4}$ developed before Proposition 8.3.1, one can sketch the graph of $\mathrm{H}$ on $\mathrm{X}_{4}$ for this choice of circulations and see that it has no critical points on $X_{4}-\Delta$.

8.4. Relative equilibria with $\sigma=0$. Recall from Chapter 1 that the relative equilibria when $\sigma=0$ are exactly the critical points of $\tilde{H}$ restricted to the set where $M=0$ in configuration space. This is a hyperplane section of $\mathbf{P}^{2}-\Delta$, that is, $\mathbf{P}^{1}-\Delta$. This set is homeomorphic to $S^{2}$ minus several points.

THEOREM 8.4.1. Let $\left(\kappa_{1}, \kappa_{2}, \kappa_{3}, \kappa_{4}\right)$ be a choice of circulations with $\sigma=0$ that is not a multiple or permutation of $(1,1,-1,-1)$. Then there are at least four relative equilibrium configurations.

Proof. Let $S^{2}$ be the subset of $N$ where $M=0$. Recall that the functions $M$ and $S$ are translation-invariant when $\sigma=0$ and $M=0$. Then observe that $S^{2} \cap \Delta_{l j} \cap$ $\Delta_{l^{\prime} j^{\prime}}=\varnothing$ if $(l, j) \neq\left(l^{\prime}, j^{\prime}\right)$ : pick the translate of $z$ that has $z_{l}=z_{j}=0$, so that $M \neq 0$ (the case $\kappa_{l^{\prime}}+\kappa_{j^{\prime}}=0$ was excluded by hypothesis). So on $S^{2},|H| \rightarrow \infty$ only at the six points $\Delta_{l j}$ and on the set $\{S=0\}$.

Without loss of generality, we may assume that $\kappa_{1}$ and $\kappa_{2}$ are positive, and $\kappa_{4}$ is negative. Consider the cases $\kappa_{3}>0$ and $\kappa_{3}<0$ separately.

If $\kappa_{3}>0$, it is easy to see from Proposition 8.3.1 that $\{S=0\} \cap S^{2}=\varnothing$. So $\tilde{H}$ is a real-valued smooth function on $S^{2}$ that is singular at six points. At $z_{l}=z_{4}$, $l=1,2,3, \tilde{H} \rightarrow-\infty$; at the other three diagonal points $\Delta_{12}, \Delta_{13}, \Delta_{23}, \tilde{H} \rightarrow \infty$. As in the preceding section, we smooth $\tilde{H}$ near these poles to find a real-valued function on $S^{2}$ that has three prescribed minima and three prescribed maxima. Morse theory then implies that $\tilde{H}$ has at least four saddle points on $S^{2}$, which are relative equilibrium configurations.

Next suppose that $\kappa_{3}<0$. By Proposition 8.3.1, $\{S=0\} \cap S^{2} \approx S^{1}$, a circle. So $\tilde{H}$ is a smooth function on $S^{2}$ that goes to $\pm \infty$ on this circle and at six other points. We will find that each component of $S^{2}-S^{1}$ contains two points where $\tilde{H} \rightarrow-\infty$, and one where $\tilde{H} \rightarrow \infty$. Given this claim, the theorem follows, for each component of $S^{2}-S^{1}$ is equivalent to a sphere $S^{2}$ with two prescribed maxima and two prescribed minima (that is, we glue a disc containing a single minimum to $S^{1}$ ). Each must then have two saddle points, relative equilibrium configurations.

To prove the claim, it suffices to show that $S$ is negative for exactly two diagonal points $\Delta_{l j} \cap S^{2}$ where $\kappa_{l} \kappa_{j}<0$, and one where $\kappa_{l} \kappa_{j}>0$. These points will then lie in one component of $S^{2}-S^{1}$; a similar statement holds for the other component.

First consider $\Delta_{12}$ and $\Delta_{34} ; \tilde{H} \rightarrow \infty$ at these points. Taking the translate of $\Delta_{12}$ with $0=z_{1}=z_{2}$, we see that $S<0$. Similarly, $S>0$ at $\Delta_{34}$. So the points at $\infty$ are evenly divided between the components of $S^{2}-S^{1}$. 
At the other diagonal points, $\tilde{H} \rightarrow-\infty$. Substituting the equation $M=0$ into the equation $S=0$, we find that the sign of $S$ at $\Delta_{13}$ is the opposite of the sign of $\left(\kappa_{2}+\kappa_{4}\right)=-\left(\kappa_{1}+\kappa_{3}\right)$, and that $S$ at $\Delta_{24}$ has sign $=\operatorname{sign}\left(\kappa_{2}+\kappa_{4}\right)$. Since $\kappa_{1}+\kappa_{2}$ $+\kappa_{3}+\kappa_{4}=0, S$ takes opposite signs at $\Delta_{13}$ and $\Delta_{24}$. A similar statement holds for $\Delta_{14}$ and $\Delta_{23}$, which proves the claim, and the theorem.

REMARK. By computing the behavior of $\tilde{H}$ near $S^{1}$ in more detail, it is possible to show that Theorem 8.4.1 holds for $\kappa=(1,1,-1,-1)$ also.

8.5. Numerical results. In Proposition 6.3.3 it was proved that the degree of a component in $\mathbf{R} P^{n-2}-\Delta$ can change under a perturbation of the choice of circulations $\kappa$ only when $\kappa$ passes through a nonregular value. Such a degree change corresponds to a collinear relative equilibrium moving onto the diagonal. That such possibilities actually do occur is demonstrated by the following computations.

Table 1 shows the position of a collinear relative equilibrium with choice of circulations $\left(\kappa_{1}, 1,1,1\right)$ as $\kappa_{1}$ approaches the value $-\frac{1}{2}$. Of course $\left(-\frac{1}{2}, 1,1,1\right)$ is not regular, for the angular momentum of the first three vortices vanishes. The first three vortices are seen to approach an equilibrium, while the last moves out to infinity. In projective space, this approaches $\Delta_{12} \cap \Delta_{23}$. The calculation was performed by fixing $z_{2}$ and $z_{3}$, and using Newton's method to solve for $z_{1}$ and $z_{4}$. The solutions in six components move toward the diagonal, so that the total number of collinear relative equilibria drops from 12 to 6 as $\kappa_{1}$ passes through $-\frac{1}{2}$.

TABLE 1.

Collinear Relative Equilibria with Circulations $\left(\kappa_{1}, 1,1,1\right)$

\begin{tabular}{cl}
\multicolumn{2}{c}{ as $\kappa_{1} \rightarrow-\frac{1}{2}$} \\
\hline$\kappa_{1}$ & $\left(z_{1}, z_{2}, z_{3}, z_{4}\right)$ \\
\hline-.3 & $(.5032,0,1,2.714)$ \\
-.4 & $(.5013,0,1,3.548)$ \\
-.47 & $(.5003,0,1,5.952)$ \\
-.49 & $(.50005,0,1,9.884)$ \\
-.499 & $(.5,0,1,30.09)$
\end{tabular}

Table 2 shows a similar phenomenon occurring as $\kappa_{1} \rightarrow-1$. Again the solutions move to $\Delta$ as $\kappa_{1}$ approaches the nonregular value. Note that the rate of rotation diverges. When $\kappa_{1}=-1$, it is easily checked that there are no collinear relative equilibria; one does this by graphing a defining function on the set $\{S=0\} \approx S^{1}$.

Families of collapsing configurations, as discussed in Chapter 7, can also be found by computation. For the 4-vortex system with $L=0$, the families lie in the set $\{S=0\} \approx S^{3}$, and appear as the common zeros of two real-valued functions. The families for a few choices of circulation are displayed in Figures 7-12, by embedding $S^{3}$ in $\mathbf{C}^{2}$ in the natural way, and then projecting onto the two factors. Circled points 
on the families are relative equilibria, and the positions in configuration space of these points are listed (to three-figure accuracy). If both complex factors are real, then the configuration is collinear.

\section{TABLE 2.}

Collinear Relative Equilibria with Circulations

\begin{tabular}{cl}
\multicolumn{2}{c}{$\left(\kappa_{1}, 1,1,1\right)$ as $\kappa_{1} \rightarrow-1$} \\
\hline$\kappa_{1}$ & $\left(z_{1}, z_{2}, z_{3}, z_{4}\right)$ \\
\hline-.8 & $(-.1844,0,1,1.636)$ \\
-.9 & $(-.0992,0,1,1.472)$ \\
-.97 & $(-.0313,0,1,1.262)$ \\
-.99 & $(-.0104,0,1,1.149)$ \\
-.995 & $(-.0051,0,1,1.104)$ \\
\hline
\end{tabular}
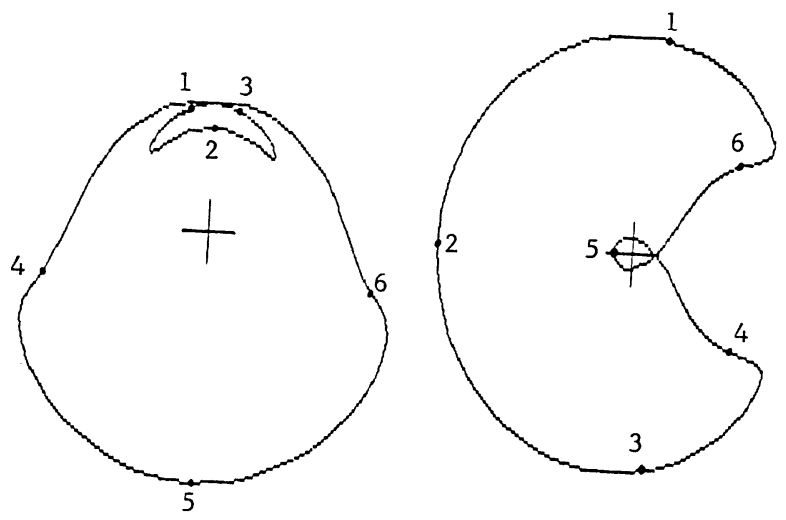

\begin{tabular}{llll} 
& \multicolumn{1}{c}{$z_{1}$} & \multicolumn{1}{c}{$z_{2}$} & \multicolumn{1}{c}{$z_{3}$}
\end{tabular}$\quad\left(z_{4}=1\right)$

FIgURE 7. Collapse family, $\kappa=(1,1,1,-1)$. 

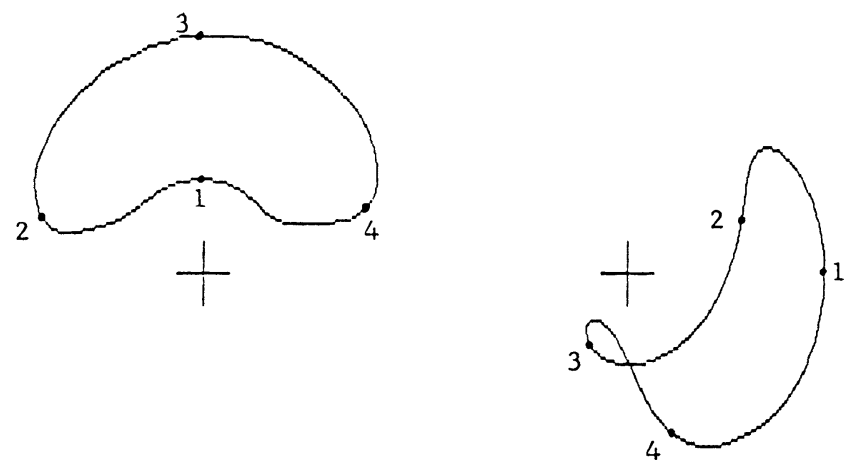

\begin{tabular}{llll} 
& \multicolumn{1}{c}{$z_{1}$} & \multicolumn{1}{c}{$z_{2}$} & \multicolumn{1}{c}{$z_{3}$} \\
1. & $(-.226,-.175)$ & $(.648,0)$ & $(-.142, .367)$ \\
2. & $(-.372,0)$ & $(.497, .09)$ & $(.759, .356)$ \\
3. & $(.410,-.602)$ & $(.213, .106)$ & $(.325, .778)$ \\
4. & $(.512,-.443)$ & $(.372, .252)$ & $(-.512,-.119)$
\end{tabular}

Figure 8. Collapse family, $\kappa=\left(1,2, \frac{1}{2},-1\right)$.
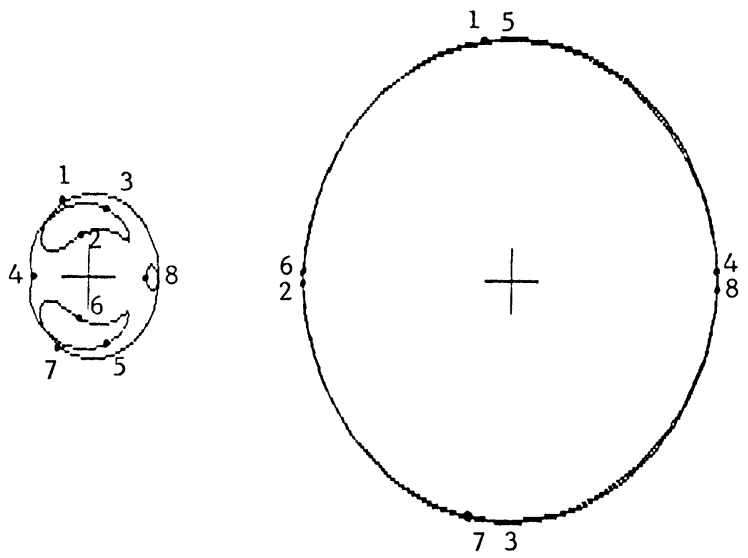

\begin{tabular}{llll} 
& \multicolumn{1}{c}{$z_{1}$} & \multicolumn{1}{c}{$z_{2}$} & \multicolumn{1}{c}{$z_{3}$} \\
1. & $(.314,-.342)$ & $(.221, .365)$ & $(.487,-.776)$ \\
2. & $(.784, .094)$ & $(-.099,0)$ & $(.829,-.154)$ \\
3. & $(.373, .636)$ & $(.243,-.371)$ & $(.279, .213)$ \\
4. & $(-.348,0)$ & $(.662,0)$ & $(.047,0)$ \\
5. & $(.399,-.631)$ & $(.228, .369)$ & $(.287,-.215)$ \\
6. & $(.776,-.075)$ & $(-.099,0)$ & $(.848, .162)$ \\
7. & $(.3, .341)$ & $(.238,-.367)$ & $(.450, .787)$ \\
8. & $(-.078,0)$ & $(.661,0)$ & $(-.449,0)$
\end{tabular}

Figure 9. Collapse family, $\kappa=\left(1,2, \frac{1}{2},-1\right)$. 

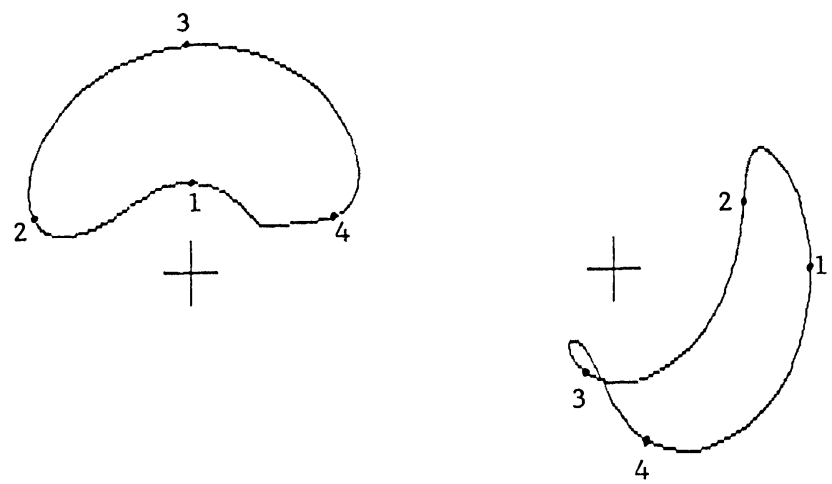

\begin{tabular}{llll} 
& \multicolumn{1}{c}{$z_{1}$} & \multicolumn{1}{c}{$z_{2}$} & \multicolumn{1}{c}{$z_{3}$} \\
1. & $(-.208, .168)$ & $(.650,-.011)$ & $(-.170,-.378)$ \\
2. & $(.499, .446)$ & $(.341,-.267)$ & $(-.544, .101)$ \\
3. & $(.328, .592)$ & $(.226,-.158)$ & $(.436,-.781)$ \\
4. & $(-.342,-.097)$ & $(.526, .1)$ & $(.754,-.347)$
\end{tabular}

FIGURE 10. Collapse family, $\kappa=(1.1,2, .4287,-1)$.
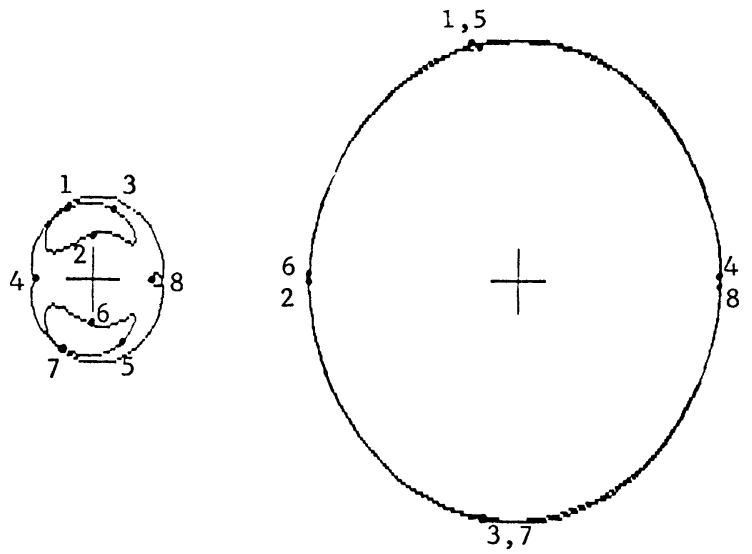

\begin{tabular}{llll} 
& \multicolumn{1}{c}{$z_{1}$} & \multicolumn{1}{c}{$z_{2}$} & \multicolumn{1}{c}{$z_{3}$} \\
1. & $(.313,-.359)$ & $(.222, .369)$ & $(.495,-.801)$ \\
2. & $(.774, .086)$ & $(-.105,-.012)$ & $(.834,-.165)$ \\
3. & $(.371, .608)$ & $(.237,-.375)$ & $(.277, .187)$ \\
4. & $(-.325,0)$ & $(.664,0)$ & $(.069,0)$ \\
5. & $(.389,-.605)$ & $(.225, .374)$ & $(.282,-.191)$ \\
6. & $(.769,-.07)$ & $(-.105,0)$ & $(.849, .170)$ \\
7. & $(.303, .358)$ & $(.233,-.371)$ & $(.466, .81)$ \\
8. & $(-.096,0)$ & $(.662,0)$ & $(-.511,0)$
\end{tabular}

FIGURE 11. Collapse family, $\kappa=(1.1,2, .4287,-1)$. 

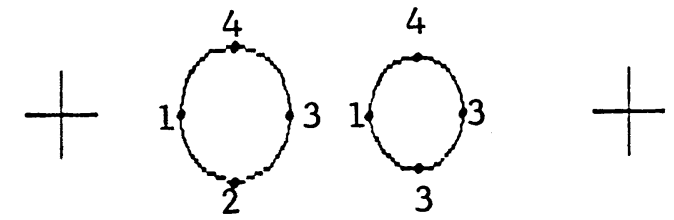

3

\begin{tabular}{lllll} 
& \multicolumn{1}{c}{$z_{1}$} & \multicolumn{1}{c}{$z_{2}$} & \multicolumn{1}{c}{$z_{3}$} & $\left(z_{4}=1\right)$ \\
1. & $(.936,0)$ & $(.334,0)$ & $(0,0)$ & \\
2. & $(.930,0)$ & $(0,0)$ & $(0, .303)$ \\
3. & $(.927,0)$ & $(-.382,0)$ & $(0,0)$ \\
4. & $(.93,0)$ & $(0,0)$ & $(0,-.292)$
\end{tabular}

FIGURE 12. Collapse family, $\kappa=(1.1,2, .4287,-1)$.

The first family (Figure 7) was computed with $\kappa=(1,1,1,-1)$. Although there are no collinear relative equilibria, this family contains six relative equilibria, which are zero crossings for the rate of collapse, $\operatorname{Re}\left(V_{l} / z_{l}\right)$. Of course, the complex conjugate of this family is also a family of collapses.

Figures 8 and 9 are the collapse families for $\kappa=\left(1,2, \frac{1}{2},-1\right)$. Note that one of these families contain no collinear relative equilibria, while the other does. In the second family, one circuit of the left-hand curve corresponds to two of the right-hand curve.

Figures 10-12 show the collapse families for $\kappa=(1.1,2, .4287,-1)$. Figure 6 predicts the appearance of two new collinear relative equilibria, which in fact lie on a new family of collapse configurations.

AcKnowledgments. I would like to thank Professor Julian Palmore for his guidance and many patient conversations.

\section{REFERENCES}

H. Aref (1983), Integrable, chaotic and turbulent vortex motion in two-dimensional flows, Ann. Rev. Fluid Mech. 15.

(1979), Motion of three vortices, Phys. Fluids 22, 393-400.

H. Aref and N. Pomphrey (1982), Integrable and chaotic motions of four vortices, Proc. Roy. Soc. London Ser. A 380, 359-387.

G. K. Batchelor (1967), An introduction to fluid dynamics, Cambridge Univ. Press, Cambridge, England, Chapter 7.

K. O. Friedrichs (1966), Special topics in fluid dynamics, Chapter 19, Gordon and Breach, New York.

P. Griffiths and J. Harris (1978), Principles of algebraic geometry, Wiley, New York.

W. Gröbli (1877), Specielle Probleme uber die Bewegung geradliniger paralleler Wirbelfaden (Zurich: Zurcher und Furrer).

T. H. Havelock (1931), The stability of motion of rectilinear vortices in ring formation, Philos. Mag. 11, 617-633.

H. Helmholtz (1858), On integrals of the hydrodynamical equations which express vortex motion, Philos. Mag., 33, 485-512.

N. Jacobson (1974), Basic algebra. I, Freeman, San Francisco, Calif.

Lord Kelvin (1910), Mathematical and physical papers, Vol. IV, Nos. 10, 12, Cambridge Univ. Press, Cambridge, England.

G. R. Kirchhoff (1876), Vorlesungen uber Matematische Physik, Vol. I, Teubner, Leipzig. 
H. Lamb (1932), Hydrodynamics, Chapter VII, Dover, New York.

E. A. Novikov (1975), Dynamics and statistics of a system of vortices, Soviet Phys.-JETP 41, 937-943.

E. A. Novikov and Yu. B. Sedov (1979), Vortex collapse, Sov. Phys.-JETP, 50, 297-301.

J. I. Palmore (1982), Relative equilibria of vortices in two dimensions, Proc. Nat. Acad. Sci. U.S.A. 79, 716-718.

I. Shafarevich (1977), Basic algebraic geometry, Springer-Verlag, New York.

A. Sommerfeld (1964), Mechanics of deformable bodies, Chapter IV, Academic Press, New York.

J. L. Synge (1949), On the motion of three vortices, Canad. J. Math. 1, 257-270.

J. J. Thomson (1883), A treatise on the motion of vortex rings, Macmillan, London.

Department of Mathematics, University of Illinois at Urbana - Champaign, Urbana, Illinois 61801

Current address: Department of Mathematics, University of Tulsa, 600 S. College Avenue, Tulsa, Oklahoma 74104 